\title{
Investigating the mobilome in clinically important lineages of Enterococcus faecium and Enterococcus faecalis
}

Theresa Mikalsen ${ }^{1 *}$, Torunn Pedersen ${ }^{2}$, Rob Willems ${ }^{3}$, Teresa M Coque ${ }^{4,5}$, Guido Werner ${ }^{6}$, Ewa Sadowy ${ }^{7}$, Willem van Schaik ${ }^{3}$, Lars Bogø Jensen ${ }^{8}$, Arnfinn Sundsfjord ${ }^{1,2+}$ and Kristin Hegstad ${ }^{1,2^{*}+}$

\begin{abstract}
Background: The success of Enterococcus faecium and E. faecalis evolving as multi-resistant nosocomial pathogens is associated with their ability to acquire and share adaptive traits, including antimicrobial resistance genes encoded by mobile genetic elements (MGEs). Here, we investigate this mobilome in successful hospital associated genetic lineages, E. faecium sequence type (ST)17 $(n=10)$ and ST78 $(n=10)$, E. faecalis ST6 $(n=10)$ and ST40 $(\mathrm{n}=10)$ by DNA microarray analyses.

Results: The hybridization patterns of 272 representative targets including plasmid backbones ( $n=85$ ), transposable elements ( $n=85)$, resistance determinants $(n=67)$, prophages $(n=29)$ and clustered regularly interspaced short palindromic repeats (CRISPR)-cas sequences $(n=6)$ separated the strains according to species, and for $E$. faecalis also according to STs. RCR-, Rep_3-, RepA_N- and Inc18-family plasmids were highly prevalent and with the exception of Rep_3, evenly distributed between the species. There was a considerable difference in the replicon profile, with rep $17 / p R$ M $_{1}$, rep $2 /$ PRE25, rep 14/EFNP1 and rep 20/pLG1 dominating in E. faecium and rep 9/PCF10, rep $_{2 / D R E 25}$ and rep 7 in E. faecalis strains. We observed an overall high correlation between the presence and absence of genes coding for resistance towards antibiotics, metals, biocides and their corresponding MGEs as well as their phenotypic antimicrobial susceptibility pattern. Although most IS families were represented in both E. faecalis and E. faecium, specific IS elements within these families were distributed in only one species. The prevalence of IS256-, IS3-, ISL3-, IS200/IS605-, IS110-, IS982- and IS4-transposases was significantly higher in E. faecium than E. faecalis, and that of IS110-, IS982- and IS1182-transposases in E. faecalis ST6 compared to ST40. Notably, the transposases of IS981, ISEfm1 and IS1678 that have only been reported in few enterococcal isolates were well represented in the E. faecium strains. E. faecalis ST40 strains harboured possible functional CRISPR-Cas systems, and still resistance and prophage sequences were generally well represented.
\end{abstract}

Conclusions: The targeted MGEs were highly prevalent among the selected STs, underlining their potential importance in the evolution of hospital-adapted lineages of enterococci. Although the propensity of inter-species horizontal gene transfer (HGT) must be emphasized, the considerable species-specificity of these MGEs indicates a separate vertical evolution of MGEs within each species, and for E. faecalis within each ST.

Keywords: Hospital associated/clinical enterococcus, Horizontal gene transfer, Mobile genetic elements, Antibiotic resistance

\footnotetext{
*Correspondence: Theresa.Mikalsen@uit.no; Kristin.Hegstad@uit.no

†'Equal contributors

'Research group for Host-microbe Interactions, Department of Medical Biology, Faculty of Health Science, UiT - The Arctic University of Norway, Tromsø, Norway ${ }^{2}$ Norwegian National Advisory Unit on Detection of Antimicrobial Resistance, Department of Microbiology and Infection Control, University Hospital of North Norway, Tromsø, Norway

Full list of author information is available at the end of the article
}

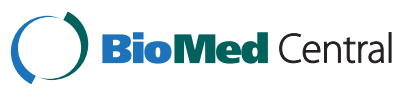

(c) 2015 Mikalsen et al.; licensee BioMed Central. This is an Open Access article distributed under the terms of the Creative Commons Attribution License (http://creativecommons.org/licenses/by/4.0), which permits unrestricted use, distribution, and reproduction in any medium, provided the original work is properly credited. The Creative Commons Public Domain Dedication waiver (http://creativecommons.org/publicdomain/zero/1.0/) applies to the data made available in this article, unless otherwise stated. 


\section{Background}

The emergence of E. faecalis and E. faecium as leading hospital pathogens worldwide has been associated with their promiscuous nature in acquiring new genetic elements through HGT. HGT facilitates the adaptation of specific genetic lineages to the hospital environment by enabling acquisition of antimicrobial resistance, bacteriocin and virulence determinants that provide selective advantages and promote gastrointestinal colonization [1-8]. Molecular epidemiological studies using multilocus sequence typing (MLST) and eBURST analysis identified a worldwide polyclonal cluster of hospital adapted E. faecium clones, termed at that time clonal complex (CC) 17, which contained sequence type (ST) 17 as well as its possible descendants; single locus variants ST16, ST78, ST63, ST64 and ST174 $[9,10]$. However, recent Bayesian-based population genetic modelling comparing whole genome sequences, suggests the existence of two clades of $E$. faecium strains (clade A and B), where clade A (A1) includes E. faecium associated with human infections from $\mathrm{CC} 17$ as opposed to clade B that contains strains of non-hospital human origin $[6,11,12]$. E. faecalis, in general, seem to be less origin- and/or host-restricted as dominant clones are shared between hospitals and the community although some CCs, including CC2, CC40 and CC87 show clear over-representation in hospitalassociated infections [13].

Whole genome sequencing, comparative genome analysis and molecular epidemiological studies have provided important information about the content and distribution of MGEs in E. faecalis and E. faecium [11,14-21]. Plasmids, transposons, and prophages all contribute to the plasticity of enterococcal genomes [2,22] and clinical E. faecium strains have twice as many genes associated with MGEs as compared to non-clinical strains [16]. Sequence variability, presence of mosaic structures of plasmids combining modules from various origin and chimeric plasmids indicates a high genetic diversity of enterococcal plasmids [21,23-26]. Further, plasmid-mediated intraspecies chromosome-to-chromosome transfer of large DNA segments has been documented for both E. faecalis [3] and $E$. faecium [27]. In hospital-associated enterococci, plasmid stabilizing toxin-antitoxin (TA) systems, including Axe-Txe and $\omega-\varepsilon-\zeta$, are prevalent $[26,28,29]$. Notably, such TAsystems are increasingly considered as targets for development of new antimicrobial agents against multidrug resistant pathogens [30-34]. The impact of prophages on enterococcal diversity is less understood, but whole genome sequencing of E. faecium strains identified prophages as a prominent source of genome diversity $[6,15,16]$.

Our epidemiological knowledge of MGEs in enterococci is limited and have mainly been based on the characterization of a restricted number of strains. The population structure of the examined strains have not been well characterized and their selection has been from a very broad range of origins and/or biased by certain characteristics such as specific antibiotic resistance mechanisms. Thus, we still have a considerable gap in our knowledge concerning the presence and relative distribution of known MGEs in different clinically relevant genetic lineages of enterococci and the potential role of these MGEs in the ecological dominance of enterococcal lineages in hospitals. In order to provide new insight in the complex mobile gene pool of E. faecalis and E. faecium (the mobilome [35]), we developed a DNA microarray with markers of enterococcal mobile genetic elements (including antimicrobial resistance genes) and CRISPR-cas elements identified so far. The arrays were hybridized with single strain genomic DNA of 40 enterococcal hospital associated strains. The mobilome profiles distinguished enterococci at species and subspecies level. The observed homogeneous hybridization pattern between E. faecium STs is in accordance with an evolutionary relatedness of ST17 and ST78 [6].

\section{Methods}

\section{Bacterial strains}

A total of 40 human E. faecium and E. faecalis strains representing four highly prevalent and clinical relevant STs; E. faecium (ST17, n = 10; ST78, n= 10) and E. faecalis (ST6, $\mathrm{n}=10$; ST40, $\mathrm{n}=10$ ), were selected. Relevant strain characteristics are given in Table 1 . Briefly, the strains originated from nine European countries (Denmark, Germany, Italy, Norway, Poland, Portugal, Spain, Sweden, and The Netherlands) and were isolated between 1992 and 2009. With the exception of one fecal strain, all were clinical strains and some have been associated with hospital outbreaks. The fully sequenced $E$. faecalis V583 [36] was included as a control to monitor the hybridization quality.

\section{DNA isolation, hybridization and data acquisition}

Single strain genomic DNA for microarray hybridization and PCR was extracted using E.Z.N.A Bacterial DNA kit (Omega Bio-Tek Inc., Norcross, GA) with the following modifications: cell walls were digested with $7 \mu \mathrm{g} / \mu \mathrm{L}$ lysozyme and $0.5 \mathrm{U} / \mu \mathrm{L}$ mutanolysin for $20 \mathrm{~min}$ at $30^{\circ} \mathrm{C}$, and DNA was eluted with $\mathrm{ddH}_{2} \mathrm{O}$. For hybridization, DNA was broken down to 100-600 bp fragments by $1 \mathrm{~min}$ sonication at $2 \mu \mathrm{m}$ amplitude. Genomic DNA $(2 \mu \mathrm{g})$ was fluorescent labeled using the Kreatech labeling kit with ULS-Cy5 according to the manufacturer's instructions (Kreatech Biotechnologies, Amsterdam, The Netherlands), and degree of labeling (DoL) was calculated by measuring absorbance at 260 and $650 \mathrm{~nm}$ using a Nanodrop spectrophotometer. Hybridization of $4 \mathrm{X} 2 \mathrm{~K}$ CustomArrays were performed as described by the manufacturer (CustomArray 
Table 1 Clinical enterococcal strains used for hybridization

\begin{tabular}{|c|c|c|c|c|c|c|}
\hline No & ST/CC & Name & Source & Country & Year & Reference \\
\hline \multicolumn{7}{|l|}{ E. faecium } \\
\hline 1 & $17 / 17$ & U0229 & Blood & NLD & 1995 & [37] \\
\hline 2 & $17 / 17$ & TUH2-18 & Urine & NOR & 1996 & {$[38,39]$} \\
\hline 3 & $17 / 17$ & U0218 & Blood & NLD & 1997 & [37] \\
\hline 4 & $17 / 17$ & E1463 & Blood & ESP & 1998 & {$[40]$} \\
\hline 5 & $17 / 17$ & O2T878 & Blood & SWE & 2002 & {$[41]$} \\
\hline 6 & $17 / 17$ & NIZP292/02 & Wound & POL & 2002 & {$[42]$} \\
\hline 7 & $17 / 17$ & U0106 & Blood & NLD & 2004 & [37] \\
\hline 8 & $17 / 17$ & VRE-10 & Blood & DEN & 2005 & [43] \\
\hline 9 & $17 / 17$ & UW6900 & Blood & GER & 2006 & {$[44,45]$} \\
\hline 10 & $17 / 17$ & VRE-84 & VA & DEN & 2008 & [43] \\
\hline 11 & $78 / 17$ & E1644 & Urine & GER & 2002 & {$[46]$} \\
\hline 12 & $78 / 17$ & U0262 & Blood & NLD & 2004 & [37] \\
\hline 13 & $78 / 17$ & U0367 & Blood & NLD & 2004 & [37] \\
\hline 14 & $78 / 17$ & E2603 & Blood & NLD & 2005 & [37] \\
\hline 15 & $78 / 17$ & E4076 & Blood & NLD & 2006 & [37] \\
\hline 16 & $78 / 17$ & UW6847 & Blood & GER & 2006 & {$[45]$} \\
\hline 17 & $78 / 17$ & UW6880 & Blood & GER & 2006 & {$[45,47]$} \\
\hline 18 & $78 / 17$ & $\mathrm{HPH} 3$ & Urine & PRT & 2007 & {$[47]$} \\
\hline 19 & $78 / 17$ & VRE-106 & Urine & DEN & 2008 & [43] \\
\hline 20 & $78 / 17$ & VRE0673 & Feces & SWE & 2008 & [48] \\
\hline \multicolumn{7}{|l|}{ E. faecalis } \\
\hline 21 & $6 / 2$ & 229710 & Urine & PRT & 1992 & [49] \\
\hline 22 & $6 / 2$ & 2724 & Blood & ITA & 1993 & {$[50]$} \\
\hline 23 & $6 / 2$ & 217691 & Blood & PRT & 1996 & {$[50,51]$} \\
\hline 24 & $6 / 2$ & E1828 & Blood & ESP & 2001 & {$[52]$} \\
\hline 25 & $6 / 2$ & E3450 & Blood & NLD & 2006 & [13] \\
\hline 26 & $6 / 2$ & UW7001 & Blood & GER & 2006 & [13] \\
\hline 27 & $6 / 2$ & $340 / 07$ & Blood & $\mathrm{POL}$ & 2007 & [13] \\
\hline 28 & $6 / 2$ & 1665/07 & Blood & DEN & 2007 & [13] \\
\hline 29 & $6 / 2$ & VRE-115 & Blood & DEN & 2008 & {$[43]$} \\
\hline 30 & $6 / 2$ & $3962 / 09$ & Blood & NLD & 2009 & [13] \\
\hline 31 & $40 / 40$ & $435 / 96$ & Urine & $\mathrm{POL}$ & 1996 & {$[53,54]$} \\
\hline 32 & $40 / 40$ & UW1833 & Urine & GER & 1998 & This study, $[53,54]$ \\
\hline 33 & $40 / 40$ & 7239/99 & Urine & POL & 1999 & {$[53-55]$} \\
\hline 34 & $40 / 40$ & HC24 & Blood & ESP & 2001 & {$[52-54]$} \\
\hline 35 & $40 / 40$ & $457 / 04$ & Urine & $\mathrm{POL}$ & 2004 & {$[53-55]$} \\
\hline 36 & $40 / 40$ & UW5744 & Urine & GER & 2004 & This study, $[53,54]$ \\
\hline 37 & $40 / 40$ & UW6756 & Urine & GER & 2006 & This study, $[53,54]$ \\
\hline 38 & $40 / 40$ & UW7790 & Feces & $\mathrm{POL}$ & 2007 & {$[53-55]$} \\
\hline 39 & $40 / 40$ & 1638/07 & Urine & DEN & 2007 & [13] \\
\hline 40 & $40 / 40$ & 3992/09 & Urine & NLD & 2009 & [13] \\
\hline Control & $6 / 2$ & V583 & Blood & USA & 1981 & {$[36,56]$} \\
\hline
\end{tabular}


Inc, Mukilteo, WA) at $52^{\circ} \mathrm{C}$ for 15 hours. Slides were subsequently scanned using an Axon GenePix ${ }^{\circ}$ 4000B scanner. Immediately after scanning, the slides were stripped for 90 min according to the manufacturer's protocol. Control scans were performed each time to monitor possible background of the subsequent re-hybridization. The slides were stripped up to 6 times.

\section{Data analysis}

E. faecalis V583 [36] was used as a technical replicate control on each fourplex array. Specifically, given that both the E. faecalis V583 genome and array probe sequences are known, number of false positive hybridizations could be monitored. A signal intensity cut-off could therefore be determined for each array, which reduces these hybridizations with more than $95 \%$. The entire dataset was then normalized using quantile normalization. The overall correlation for all technical replicates included in study was 0.95, (see the correlation matrix for quantile normalized technical replicates in Additional file 1: Table S1). A hierarchical cluster diagram of hybridization data made in $\mathrm{R}$ calculated the distance by the method "complete". Principal component analyses (PCA) and a cluster dendrogram were run on the resultant quantile normalized matrix (see Additional file 2: Figure S1A and B, respectively). The presence or absence of each target in each of the 40 clinical E. faecium ST17 (n= 10) and ST78 $(\mathrm{n}=10)$ and E. faecalis ST6 $(\mathrm{n}=10)$ and ST40 $(\mathrm{n}=10)$ is presented in Additional file 3: Figure S2A-E. Each target is represented as a mean value of its 1-5 probes. Number of probes and probe sequences are listed in Additional file 4: Table S2.

\section{Probe design and final target list}

The bioinformatics analysis and probe design of initially 405 targets sequences was done by CustomArray support (http://customarrays.com/index.htm) (last accessed December $\left.10^{\text {th }}, 2014\right)$. A total of 133 targets were excluded due to sequence similarity $(>90 \%)$, sequence quality, and misleading annotation in GenBank. For the remaining 272 target sequences, 1-5 probes were designed. Suggested probes were blasted against a database built of the following enterocccal genome sequences: (E. faecium DO (GenBank acc. no. AAK03000000), E. faecium strains E1039 (NZ_ ACOSO1000000), E1071 (NZ_ABQI00000000), E1162 (NZ_ABQJ00000000), E1636 (NZ_ABRY00000000), E1679 (NZ_ABSC00000000), E980 (NZ_ABQA00000000), and U0317 (NZ_ABSW01000000), and the E. faecalis strains OG1RF (CP002621), TX0104 (NZ_ACGL00000000), and TX1322 (NZ_ACGM00000000). Probes with most homologues (increased probability of false positive hybridizations) were excluded. The resulting 1250 probes were Tm-balanced by altering their length between 35 and 40 nucleotides. Number of final probes for each target and probes sequences are given in Additional file 4: Table S2.

The final target list included plasmid backbone genes $(n=85)$ encoding replication initiation proteins, genes associated with plasmid conjugation maintenance and plasmid addiction systems; transposable elements $(n=85)$ including conjugative transposons (integrases, excisionases and relaxases), Tn3 family of transposons (transposases and resolvases), transposases associated to known IS elements, and other targets associated to transposable elements; genes encoding resistance $(n=67)$ towards relevant antibiotics (glycopeptides, aminoglycosides, $\beta$-lactamases, tetracyclines, macrolides/lincosamides/streptogramins $\mathrm{B}\left(\mathrm{MLS}_{\mathrm{B}}\right.$ antibiotics), linezolid, chloramphenicol, and trimethoprim), biocides (disinfectants), and heavy metals; prophage sequences $(\mathrm{n}=29)$ and CRISPR-cas sequences $(n=6)$. Thirty six plasmid replicon variants associated with enterococci were included in the analyses. Previously defined 20 reps $[21,24]$ are designated by subscripted number representing replicon type and/or the name of the reference plasmid in Additional file 5: Figure S3A.

\section{PCR}

The presence of important targets rejected during the probe design or targets representing newly described mobile genetic elements were examined by specific PCRs. They included $a a c\left(6^{\prime}\right)$ - $a p h\left(2^{\prime \prime}\right)$-Ia encoding the bi-functional aminoglycoside-modifying enzyme [57], the $v a n A$ and $v a n B$ clusters $[38,58,59]$ as well as genes encoding the replication initiation proteins of plasmids pLG1 [29,60], pCF10 [24] and pIP501 (repR) [24]. To detect both $\omega-\varepsilon-\zeta$ phylogenetic subgroups [17], the following primer pair specific for conserved regions were used: EU2f: 5'-GGCGGAAACGTAAAA GAAGTTATG-3' and EU4r: 5' -TTCATTGACCGC CAATACTCATG-3'.

\section{Antimicrobial susceptibility testing}

Antimicrobial susceptibility testing (AST) of the strains was performed to examine the validity of the microarray hybridization results for defining antimicrobial resistance determinants. AST towards ampicillin $(2 \mu \mathrm{g})$, erythromycin $(15 \mu \mathrm{g})$, gentamicin $(30 \mu \mathrm{g})$, linezolid $(10 \mu \mathrm{g})$, and tetracycline $(30 \mu \mathrm{g})$ were done according to EUCAST (European Committee for Antimicrobial Susceptibility Testing) disk diffusion method [61]. Vancomycin susceptibility was examined using the brain heart infusion (BHI) agar screen method [62] using BHI supplemented with $6 \mathrm{mg} / \mathrm{L}$ vancomycin. The EUCAST clinical breakpoints [63] or clinical breakpoints defined by Clinical and Laboratory Standards Institute (CLSI) for antimicrobials not defined by the EUCAST were used for interpretation of AST. 


\section{Statistical calculations}

The statistical differences in hybridization profile of selected targets between either the two species (E. faecium/E. faecalis) or between STs within the two species (ST17/ST78 or ST6/ST40, respectively) were assessed by chi square $\left(\chi^{2}\right)$ calculations without corrections and with 1 degree of freedom using the online calculator http://graphpad.com/ quickcalcs/contingency1/ (last accessed December $10^{\text {th }}$, 2014). P-value $<0.05$ was considered significant.

\section{Results and discussion}

Clinical relevant strains of European origin belonging to four predominant STs from the two decades of 1990 and 2000 were chosen for this study; E. faecium ST17 and ST78 which represent Bayesian Analysis of Population Structure (BAPS) groups $3-3$ and $2-1$ and E. faecalis ST6 and ST40 of CC2 and CC40, respectively. The DNA microarray was designed to cover mobile genetic elements (including antimicrobial resistance determinants) and CRISPR-cas elements associated with enterococci and known at that time.

\section{Overall hybridization profile and quality}

A PCA was applied on the overall dataset and visualized in a two-dimensional plot (see Additional file 2: Figure $\mathrm{S} 1 \mathrm{~A}$ ), also a dendrogram was created to visualize the result of a hierarchical clustering calculation of the mobilome (see Additional file 2: Figure S1B). The overall hybridization pattern showed E. faecium ST17 and ST78 clustered while the E. faecalis ST6 and ST40 formed two valid subgroups. As these data covers genes mainly involved in HGT and to a much less extent vertical descent, this does not describe the clonal relatedness of the strains analyzed. MLST-based data show that E. faecium ST17 and ST78 belong to two different BAPS groups (see above) suggesting that both STs are part of different hospital lineages and followed different evolutionary trajectories [64]. Although analyzing different strains than ours, the phylogenetic distance between E. faecium ST17 and ST78 is partly addressed through WGS by Lebreton et al. showing these strains grouped in one clade (clade A1) [6]. Within this clade, however, ST17 and ST78 cluster in different lineages, suggesting a certain degree of common evolutionary background yet diversification into separate genetic lineages. The highly-common mobilome found in our E. faecium ST17 and ST78 strains is most likely the result of sharing a common ecological niche (hospital) in which a common set of accessory genes is necessary to survive and thrive rather than evolutionary relatedness. Complete relatedness can be inferred by next generation sequencing (NGS), however, this is not the scope of our study. The DNA microarray technique was preferred over NGS as we searched only for absence or presence and not localization of specific targets. Also, reliable de novo whole genome assemblies from Illumina data addressing the mobile genome content is limited [65-67]. The dots representing every application of the control strain, E. faecalis V583 (ST6) form a tight cluster ensuring the comparable quality of the slides. The PCA shows that the selected targets in this array are both species- and ST-specific. Thus, the overall results of the PCA indicate that the dataset is technically accurate and biologically relevant. The accuracy of each repeat of the hybridization was also monitored by correlating the overall hybridizations of the technical replicate E. faecium V583 included in each fourplex array (Additional file 1: Table S1 and Materials \& Methods). A correlation of 0.95 was obtained. As an additional quality control of the array, the presence and absence of antimicrobial resistance markers were compared with antimicrobial susceptibility testing and by selected consensus PCR analyses. This revealed that the results from the verifications were in agreement with the microarray hybridization results close to the expected $95 \%$ accuracy (data not shown).

\section{Species- and ST-specific patterns of mobile genetic elements}

The detailed MGE gene profile of the 40 sample strains is given in Additional file 3: Figure S2A-E. The data are summarized for each of the four STs in Additional file 5: Figure S3A-E, significant differences $(\mathrm{p}<0.05)$ between species and STs are indicated. The major species and ST-specific patterns are categorized and discussed below in relation to difference in content of plasmids, transposable elements, antimicrobial resistance determinants, prophages and CRISPR-cas modules.

\section{Frequently detected plasmid associated gene targets}

Plasmid-encoded genes involved in conjugation and mobilization as well as replication and maintenance were assessed by microarray hybridization analyses or, for a few targets, by PCR (Additional file 3: Figures S2A and Additional 5: Figure S3A). In order to sort out biological relevant patterns within the complexity caused by the modular structure of plasmids and the propensity of inter-plasmid recombination events, we defined the plasmid content in the four STs by presence of rep genes encoding replication initiator proteins and TA-encoding genes.

The average number of detected reps per strain for E. faecium and E. faecalis ( $\mathrm{n}=6.75$ and $\mathrm{n}=5.45$, respectively) were higher than previously reported for invasive E. faecium (3.2; [29]) and for clinical isolates of E. faecalis $(2.54 ;[26])$. This might reflect higher plasmid content in the selected STs, but may also be due to the higher number of rep targets included in the array. We suppose the factual number of plasmids to be lower than the identified rep genes since multi replicon plasmids and 
plasmid remnants in the core chromosome are known for many bacteria including enterococci $[21,48]$.

As shown in Additional file 5: Figure S3A, rep targets belonging to the RCR, Rep_3, RepA_N, and Inc18 plasmid families (as defined by [68]) were well represented in both E. faecium ( $\mathrm{n}=34 ; \mathrm{n}=28 ; \mathrm{n}=36$; and $\mathrm{n}=21$, respectively) and E. faecalis $(\mathrm{n}=27 ; \mathrm{n}=6 ; \mathrm{n}=36$; and $\mathrm{n}=28$, respectively). With the exception of the Rep_3 family (see below), there were no significant differences between the species or between the STs for the included strains. However, the composition of replicon types (as defined by [24]) within each plasmid family differed highly between $E$. faecium and E. faecalis and for some rep types between the STs as indicated. Our findings are in accordance with previous reports, although the composition and prevalence of the reps appear to vary between strain collections of different origins $[17,21,26,29]$.

The rep $_{14}$ belonging to the RCR family was the most abundant replicon type in the strain collection with a significant $(\mathrm{p}<0,0001)$ higher prevalence in E. faecium $(\mathrm{n}=32)$ than in E. faecalis $(\mathrm{n}=8)$. The rep $_{14}$ replicons, which include the small mobilizable plasmids pEFNP1, pKQ10 and pRI1, have been considered specific for $E$. faecium [24,68-70]. Sequences originating from pEFNP1 and $\mathrm{pKQ} 10$ were prominent in a hospital clade specific E. faecium plasmid library [14]. Interestingly, the rep of pEFNP1 (target 19) was also found in half of the E. faecalis ST40 strains included in our study. Plasmids of rep $_{7 / p U S A O 2}$ type have been obtained from a broad range of hosts (Enterococcus, Staphylococcus, Streptococcus and Bacillus) and were represented in the array (target 431) by a repD of an E. casseliflavus plasmid [71]. Strikingly, the rep $_{7}$ replicons were found in 12/20 of the E. faecalis strains, but only in two E. faecium strains $(\mathrm{p}<0.0001)$. Taken together, the replicons of the RCR family appear to be abundant with a species-specific profile. Nevertheless, our observations support the notion that the examined STs of E. faecium and E. faecalis could share a pool of cryptic small plasmids through HGT. Their potential role of in the evolution of clinically important lineages of enterococci remains to be examined.

Rep_3 family includes several plasmids previously described in enterococci [72-75]. In our strain collection, a significant higher prevalence of Rep_3 family replicons were detected in $E$. faecium $(\mathrm{n}=28)$ compared to $E$. faecalis $(\mathrm{n}=6 ; \mathrm{p}<0.0001)$. In E. faecium represented by $\operatorname{rep}_{18}(\mathrm{n}=11), \operatorname{rep}_{11}(\mathrm{n}=10)$ and $\operatorname{rep}_{p C I Z 2}(\mathrm{n}=7)$, the two latter have a higher prevalence in ST78 compared to ST17 ( $\mathrm{p}=0.0377$ and $\mathrm{P}=0.0191$, respectively). In E. faecalis, Rep_3 plasmids were represented by rep $5 / p N 315(\mathrm{n}=2), \operatorname{rep}_{6 / p S 86}(\mathrm{n}=2)$ and $\operatorname{rep}_{11 / p B 82}(\mathrm{n}=2)$. This highly species-specific distribution of replicons indicate a narrow host range profile of the Rep_3 family. Their high abundance in the $E$. faecium strains compared to $E$. faecalis, might reflect a specific role of this plasmid family in the development of these important clinical lineages of E. faecium.

RepA_N is the most prevalent plasmid family, but with significant differences in rep types between the two species for the included strains. This is in accordance with the description of RepA_N replicons as narrow host range plasmids [76]. The rep, variants of pheromone responsive plasmids [36,77-79] considered specific for $E$. faecalis were frequently detected in these strains but were absent in the E. faecium strains ( $\mathrm{p}<0.0001)$. Positive PCR results for $\operatorname{prgW}$ of pCF10 were observed in all E. faecalis ST6 strains. The par genes, encoding toxin RNAI and antitoxin RNAII originally found on the pheromone responsive plasmid pAD1 in E. faecalis [80], were found in $10 / 20$ of the E. faecalis strains, were also absent in the E. faecium strains $(\mathrm{p}<0.0001)$. On the other hand, the rep $17 / p R U M$ and rep $_{20 / p L G 1}$ replicons were present in $18 / 20$ and $14 / 20$ of the E. faecium strains, respectively, but absent in E. faecalis $(\mathrm{p}<0.0001)$. rep $17 /$ pRUM was the single most dominating replicon type among the selected $E$. faecium strains. This is consistent with previous reports, which also link the axe-txe TA-loci to this replicon type $[17,21,41,48,81]$. Both the axe and the txe targets (457 and 458) hybridized to $15 / 20$ E. faecium strains, while none of the E. faecalis strains were positive $(\mathrm{p}<0.0001)$. Moreover, co-hybridization with the axe-target was observed for 17/18 of the rep 17/pRUM positive and 12/14 rep $20 / p L G 1$ positive (data not shown) E. faecium strains. Also for rep $20 / p L G 1$, our findings are in agreement with previous investigations, where this rep was detected in $90 \%$ of E. faecium blood culture isolates [26] and linked to axe-txe on large conjugative plasmids encoding high-level gentamicin resistance [26] or glycopeptide resistance $[19,50,60]$. Our confirmatory findings support the importance of $r e p_{20 / p L G 1}$ megaplasmids in the evolution of hospital-associated lineages of E. faecium. Moreover, the contribution of axe-txe for the stable maintenance of clinical important RepA_N family plasmids in $E$. faecium is underlined.

The broad host range Inc18 family of conjugative plasmids is known to carry multiple antimicrobial resistance genes, including specific $\mathrm{MLS}_{\mathrm{B}}$-resistance determinants hosted by streptococci, lactococci, staphylococci, and enterococci [82]. The pRE25 replicon type $\left(\right.$ rep $\left._{2}\right)$ had the same prevalence in E. faecium and E. faecalis (14/20), while $r e p_{1}$, which includes reps from pAM $\beta 1$ and pIP501, was detected in $6 / 20$ and $7 / 20$ of the E. faecium and E. faecalis strains, respectively. No significant interspecies-differences in distribution of rep types between the included species or STs was detected, except for rep$_{p H T \beta}(\mathrm{n}=5)$, which had a higher presence in ST6 $(\mathrm{n}=4 ; \mathrm{p}=0.0233)$, which underlines the broad host range of the Inc18 plasmid family and their propensity to support genetic exchange between the two species. However, Inc18 plasmids may have been 
established in enterococci at an early stage and essentially propagated by vertical descent.

The $\omega-\varepsilon-\zeta$ TA-sequences included in the array originated from the Inc18 pVEF plasmids [83,84]. A discrepancy in presence between the three targeted TA-genes, which can be partly explained by allelic variation in the operon [17] or presence of the targets in other genetic contexts, was observed. However, to function as a plasmid stabilizing system, the presence of all three gene products is essential [85]. An additional PCR analysis using primers directed towards regions conserved for the two $\omega-\varepsilon-\zeta$ phylogenetic subgroups [17] detected the TA-locus in a total of 14/20 of E. faecium and 17/20 of E. faecalis, where all ST40 strains were positive. In agreement with previous findings [17], co-hybridization to rep $_{2 / p R E 25}$ was observed for E. faecium $(11 / 14)$ and for $E$. faecalis ST6 (6/7), confirming the linkage of $\omega-\varepsilon-\zeta$ to Inc18 plasmids in gram-positive pathogens and their contribution in stable persistence of clinically important plasmid encoded resistance traits (reviewed by [31]). However, $\omega-\varepsilon-\zeta$ was also detected in the absence of rep $_{2 / p R E 25}$ in both E. faecium $(\mathrm{n}=3)$ and E. faecalis $(\mathrm{n}=6)$ indicating linkage to other replicon types as well. Indeed, co-localization with rep 9 belonging to the RepA_N family of pheromone responsive plasmids was reported for E. faecalis [26].

The mazEF originally observed on the Escherichia coli chromosome is one of the best-characterized TAsystems and have been identified in a large number of bacterial species [86,87]. For Enterococcus, the E. coli mazEF sequence has been reported to be ubiquitously present on vanA-plasmids in VRE strains including $E$. faecium and E. faecalis [28]. However, the E. coli mazEF could not be detected in an epidemiologically diverse collection of E. faecium strains [17] and BLAST search (http://www.ncbi.nlm.nih.gov/pubmed; last accessed December $10^{\text {th }} 2014$ ) among draft genomes and plasmids did not show its presence in Enterococcus. In the current study the sequences from E. faecalis EnGen0297 strain HH22 (ACIX01000197.1) annotated as putative MazE and MazF were included (targets 464 and 465). All $E$. faecalis strains $(\mathrm{n}=20)$ were positive for both targets, while the mazEF targets were not found in the E. faecium strains $(\mathrm{p}<0.0001)$. BLAST search (last accessed December $10^{\mathrm{h}}$ 2014) for the mazEF target DNA revealed a high prevalence in $E$. faecalis genomes where it appeared to be highly conserved, while no DNA homology was found in the E. faecium genomes, which is in accordance with our findings. However, conserved ORFs encoding putative MazF homologues were prevalent among the E. faecium draft genomes, indicating evolutional segregation of the two species. The functionality and linkage of these mazEF genes to the mobilome of Enterococcus need to be further investigated.

\section{Widely distributed transposable elements}

In addition to plasmids, three groups of transposons have been shown to facilitate flux of antimicrobial resistance determinants in enterococci [2]. Composite transposons transpose accessory DNA due to homologous flanking IS elements. Moreover, the Tn3 family of transposons encode replicative transposition, and conjugative transposons support their own transfer between bacteria [2]. Our mobilome array included relevant members from all these three groups previously shown to be associated with enterococci. The IS elements belonged to families described by Clewell [68] and IS Finder (http:// www-is.biotoul.fr/) (last accessed December $10^{\text {th }}, 2014$ ).

\section{Different transposable elements in $E$. faecalis and $E$. faecium}

A total of 12 IS families and 9 transposon groups were represented in our array. Additional file 3: Figure S2B shows the gene profile of all targets included in the microarray, which represents in many cases sequences of several transposases of the same IS family. Representative targets and their distribution are summarized in Additional file 5: Figure S3B, illustrating the predominance of transposable elements in E. faecium investigated clones $(\mathrm{p}<0.0001)$. However, most of the selected targets represented transposable elements from this species and thus the relative overrepresentation of these targets among E. faecium is not unexpected. In particular, transposases of the IS families IS256 ( $\mathrm{p}=0.0026)$, IS3 ( $\mathrm{p}<$ $0.0001)$, ISL3 $(\mathrm{p}<0.0001)$, IS200/IS605 $(\mathrm{p}=0.0058)$, IS110 ( $\mathrm{p}=0.039), \quad$ IS982 $(\mathrm{p}<0.0001)$, and IS4 $(\mathrm{p}=$ $0.0079)$ were significantly more abundant in E. faecium than E. faecalis. None of the included IS families were found at higher frequencies in E. faecalis strains than in the E. faecium strains although 10 of the 11 IS families represented in E. faecium were also found in E. faecalis. The presence of IS families in both E. faecalis and E. faecium specific strains imply that these elements are spread by HGT. However, particular IS elements are distributed in only one species suggesting that these IS elements have evolved over time within this species to become different from the other IS family members. Many variants of the Tn916 family conjugative transposons have been reported (for review see [22]) and BLAST searches (last accessed December $10^{\text {th }} 2014$ ) show that both the original Tn916 and most of the variant transposons are reported in E. faecalis. For both reasons it is plausible that the conjugative transposon Tn916/Tn1545 was observed more frequently in the investigated strains of E. faecalis ( $<<0.0001)$ than E. faecium (Additional file 5: Figure S3B). Furthermore, Tn916 have also been associated with pheromone responsive plasmids in E. faecalis $[88,89]$, which accelerates their transfer among E. faecalis. 


\section{Abundant IS families and their possible association with resistance genes and transposons}

The IS256 family members (3 IS256 variants as well as IS1542, IS16, ISEf1 and IS1310) are important components of many composite transposons conferring antimicrobial resistance such as Tn5281 and Tn4001 encoding HLGR [90], Tn1547 (encoding vancomycin resistance $[91,92]$ ) and Tn5384 (encoding erythromycin and gentamicin resistance [93]. Integrated IS256-like elements have also been observed in the $\operatorname{Tn} 3$ family (Tn1546) $[23,49,94])$ and conjugative transposons (Tn5382) [39]. With the exception of IS1542 and IS1310, the IS256 family members in this study are found in the majority of the E. faecium strains $(13 / 20$ to $20 / 20)$ and in $7 / 20$ to $20 / 20$ E. faecalis strains. The presence of ISEf1, IS16 and IS1542 (target 134, 98 and 102) [49], which is often observed among $\operatorname{Tn} 1546$ variants does not correlate with the presence of this transposons (see Additional file 3: Figure S2B and Additional file 5: Figure S3B for details).

A study of $E$. faecium strains from different niches using comparative genomic hybridization (CGH) indicated an IS-driven diversification of hospital-adapted $E$. faecium strains [14]. Notably, IS16 was suggested as the most hospital clade-specific marker in E. faecium (with $98 \%$ sensitivity and 100\% specificity). This observation was further supported by detecting IS16 in 155 of 160 invasive E. faecium strains, in contrast to only three positive for this elements among 100 E. faecium strains of human commensal, animal or food-associated origin [45]. All our clinical E. faecium strains were positive for IS16, while only three $E$. faecalis strains contained this sequence.

The IS3 family was represented in this array by ISEnfa3, IS1485, IS3-like, and IS981 transposases. IS981, which is mostly reported in lactococci, was found in 17/ 20 E. faecium strains and $12 / 20$ E. faecalis strains. IS1485, which is widely spread in many enterococcal species [95], were found in all tested strains. The putative transposase of ISEnfa3 was found in all E. faecium, while it was absent in E. faecalis (Additional file 3: Figure S2B and Additional file 5: Figure S3B). ISEnfa3 was described as an epidemiological marker for Tn5382 in South Korean E. faecium [96]. However, only three ST17 E. faecium strains were positive for Tn5382, thus ISEnfa3-like elements do not seem to be associated with Tn5382 in our strains.

The ISL3 family represented by transposases from ISL3, ISL3-like, IS1251, ISEfa11, IS1476 and IS1167 dominated in the E. faecium strains with the exception of two ISL3-like transposons (see Additional file 3: Figure S2B and Additional file 5: Figure S3B for details). The presence of IS1251, ISEfa11 and IS1476 transposase sequences which were reported integrated in Tn1546-like elements [97-101] did not correlate with presence of Tn1546 (see Additional file 3: Figure S2B and Additional file 5: Figure S3B for details).
The abundance of the IS200/IS605 family in E. faecium was due to ISEfa4 and an E. faecium DO IS605 transposase, while an IS200 transposase from E. faecalis V583 was only found in E. faecalis (Additional file 3: Figure S2B and Additional file 5: Figure S3B). A putative ISEnfa200 transposase described previously as being integrated in the $v a n B$ cluster of $\operatorname{Tn} 5382$ [93] was not found in any of these strains. ISEfa4 was found in a vanS gene of a vanD genotype VRE strain [102] as well as within a Tn1546 element [103-105]. However, in our strains ISEfa4 presence did not correlate with the presence of Tn1546 (Additional file 5: Figure S3B).

The IS110 family represented by three transposases was also significantly more abundant in E. faecalis ST6 than ST40 strains $(\mathrm{p}=0.0027)$. This was due to the presence of IS110 target 165 transposase in ST6 while IS110 target 167 transposase dominated in E. faecium (Additional file 3: Figure S2B and Additional file 5: Figure S3B). The third transposase representing the putative ISEnfa110 described previously integrated in Tn5382 [92] was not found in any of these strains.

ISEfm1 representing the IS982 family has only been reported in a $v a n D$ operon of a vancomycin resistant $E$. faecium strain [106]. This IS element was present in all the E. faecium isolates and was also significantly more frequent in E. faecalis ST6 than ST40 strains ( $\mathrm{p}=0.025)$ (Additional file 3: Figure S2B and Additional file 5: Figure S3B).

The IS4 transposase can be found in many enterococcal genomes, mostly in E. faecium, This is in accordance with our results, where it was found in 6/20 E. faecium strains only.

The IS1182 family represented by IS1182 transposase is more numerous in E. faecalis ST6 than ST40 strains ( $\mathrm{p}=0.0034)$ and is also present in $7 / 20$ E. faecium strains (Additional file 3: Figure S2B and Additional file 5: Figure S3B). This IS element delimits the streptococcal composite transposon Tn5405 carrying resistance to aminoglycoside-streptothricin (aadE-sat4-aphA-3) [107]. In multiple-resistant $E$. faecium Tn5405-variants were also found genetically linked to the $\mathrm{MLS}_{\mathrm{B}}$ determinant ermB and in one case also to the glycopeptide resistance cluster vanA [108]. In concordance with this, IS1182 was in this study observed to be present only in strains containing sat4, aphA-3/aph(3)-IIIa and ermB/erm2 (targets 247, 381 and 298, respectively). However, these resistance genes were observed also in strains lacking IS1182 (Additional file 3: Figure S2B).

Due to IS1678, the IS1380 family also represented by an ISEcp1-like transposase, was statistically more prevalent in ST78 than ST17 E. faecium (p < 0.0001) (Additional file 3: Figure $\mathrm{S} 2 \mathrm{~B}$ and Additional file 5: Figure S3B). IS1678 has previously only been reported twice integrated in Tn1546 [109] and close to Tn1546 on a rep $_{17 / p R U M}$ replicon [110] in E. faecium. These IS1678 elements were, 
except for two strains, not associated with Tn1546 containing strains and detected in only $7 / 18$ E. faecium strains containing the rep ${ }_{17 / p R U M}$ replicon in our study.

\section{Predominant transposable elements encoding clinically relevant antimicrobial resistance}

To visualize the impact of resistance markers on the overall dataset, a second PCA excluding data from the targets encoding resistance markers was built (data not shown). This did not alter the overall plot substantially suggesting the resistance markers to be generally genetically linked to the other included MGEs.

The $\operatorname{Tn} 916 / \operatorname{Tn} 1545$ family of conjugative transposon (CTn) is widespread, but primarily observed in the Firmicutes such as enterococci. This CTn-family seems to have a particular ability to acquire accessory genes including resistance determinants, to cause genome rearrangements and to mobilize other replicons. The originally described Tn916 carries the tetracycline resistance gene tetM. Tn1545 is homologous to Tn916, but contains additionally ermB (encoding resistance to $M_{L} S_{B}$ antibiotics) and aphA-3 (encoding resistance to kanamycin) cassettes (reviewed in [111,112]). In our array the $\operatorname{Tn} 916 / \operatorname{Tn} 1545$ family was represented by an integrase (target 199), an excisionase (target 213) and a conjugative transfer protein gene (target 75 ) as well as the tetracyclin resistance determinant tetM (target 243). The integrase, excisionase and conjugative transfer protein genes were significantly more abundant in E. faecalis than E. faecium $(\mathrm{p}<0.0001)$ as well as in ST17 than ST78 ( $\mathrm{p}=0.02)$ of E. faecium (see Additional file 3: Figure S2B and Additional file 5: Figure S3B for details). All E. faecalis strains hybridized to the Tn916 targets while the Tn916 specific integrase and excisionase were detected in only 2/10 ST17 and were absent in ST78 E. faecium. The esp-containing ICEEfm 1 has a conjugation module similar to $\operatorname{Tn} 916$ [113]. However, the conjugative transfer protein from this conjugation module shows only about $80 \%$ identity at protein level to the Tn916 prototype protein (ORF16 target 75) and thus the DNA identity level was far too low to detect ICEEfm1 through the Tn916 conjugation protein in our array.

The Tn 3 transposon family transposon Tn1546 is genetically linked to the vanA resistance cluster encoding high-level glycopeptide resistance [114]. In our array target 146 represents the transposase [83,115], target 216 the associated resolvase [83,114] and target 237 the prototypical D-ala:D-lac ligase (vanA) of Tn1546 [115], which were present in the same strains except for vanA which was found in an additional ST17 strain (Additional file 3: Figure S2B and Additional file 5: Figure S3B). PCR (vanA) and phenotypical analysis (vancomycin) confirmed the hybridization patterns (Additional file 5: Figure S3C and data not shown).
The conjugative transposon $\operatorname{Tn} 5382 / \operatorname{Tn} 1549$ is closely linked to the vanB2 gene cluster $[92,116,117]$. This transposon was represented in our array by probes targeting the genes of the excisionase of $\operatorname{Tn} 1549$ (target 454), the integrase (target 452), the relaxase (target 39), the TrsE-like protein (target 82 ), and the D-alanine:Dlactate ligase ( $v a n B$; target 238) (Additional file 3: Figure S2B and C, Additional file 5: Figure S3B and C). A moderate number of the E. faecium and E. faecalis strains hybridized to these targets. PCR analyses and antimicrobial susceptibility testing confirmed that $\operatorname{van} B$ was present only in isolates containing all $\operatorname{Tn} 5382 / \operatorname{Tn} 1549$ related targets.

\section{Broad content of genes encoding antimicrobial resistance}

The enterococcal STs in this study were selected due to their relative dominance in clinically relevant strains. Although the presence of resistance traits was not a selection criterion, the collection is biased towards antimicrobial resistance and an overrepresentation of these markers was expected. The phenotypic expression of resistance, examined by standardized AST, was used to validate the hybridization results for defined antimicrobial resistance determinants. Moreover, the presence of $\operatorname{van} A, \operatorname{van} B$ and $\operatorname{aac}\left(6^{\prime}\right)$-aph(2")-Ia (determining high-level gentamicin resistance) was also examined by PCR.

The hybridization results of screening for the antimicrobial resistance determinants are shown in Additional file 3: Figure S2C and summarized in Additional file 5: Figure $\mathrm{S} 3 \mathrm{C}$. The results are categorized according to genes encoding resistance towards glycopeptides, aminoglycosides, $\beta$-lactams, tetracyclines, $\mathrm{MLS}_{\mathrm{B}}$, oxazolidinone, chloramphenicol, trimethoprim, biocides and heavy metals. As shown by PCA and cluster dendrogram (see Additional file 2: Figure S1A and B) a separation in gene content between the two species and the two STs of E. faecalis can be seen. Defined resistance markers were selected for phenotypic verifications, and we observed an overall high correlation as outlined below.

Vancomycin resistance determinants were represented by six targets ( 237 for $v a n A ; 238$ for $v a n B ; 280$ for vanE; 281 for $v a n G ; 284$ for $v a n G_{2} ; 313$ for $\left.v a n L\right)$. A total of 3/10 ST17 and ST78 E. faecium and ST6 E. faecalis strains were positive for the vanA target (Additional file 5: Figure S3C). The $v a n B$ target scored positive in $2 / 10$ ST17 and ST78 E. faecium strains. The vanG and vanL targets scored positive only in one and two E. faecalis ST40 strains, respectively (Additional file 5: Figure S3C). Phenotypic testing was in agreement with the hybridization results except for the vanG and vanL target positive ST40 strains (data not shown). A total of 16/40 strains expressed vancomycin resistance of which ten and six strains were confirmed as positive for $v a n A$ or $v a n B$ by PCR, respectively. As compared to $v a n A$-, $v a n B$ - and $v a n G$-specific 
PCRs, the microarray hybridization results yielded two false negative results (one vanA and one vanB E. faecium) and two potential false positive results (one vanA E. faecium and one vanG E. faecalis). Phenotypically silent vanA-determinants have previously been reported [118-120].

The presence of aminoglycoside resistance determinants showed species and ST-specific patterns. The E. faecium species-specific target $a a c\left(6^{\prime \prime}\right)-I i$ (target 254) encoding an aminoglycoside $6^{\prime}$-N-acetyltransferase that mediates resistance towards many aminoglycosides except gentamicin was present in all E. faecium strains (Additional file 5: Figure S3C). Target 247, 383 and 381 representing sat4, aadE and aph(3)-IIIa, respectively, were present in at least 10/20 strains of both E. faecium and E. faecalis (Additional file 5: Figure $\mathrm{S} 3 \mathrm{C}$ ). Other targets representing aminoglycoside resistance determinants were only sporadically present. The gene cluster aadE-sat4-aphA-3 has been linked to composite transposon Tn5405 delimited by IS1182 [107]. All three resistance genes were found in 21/40 of the isolates (Additional file 5: Figure S3C) although aadE was additionally present in four, sat4 in one and aphA-3 in three isolates. IS1182 was present in 13/21 of the isolates that contained all three resistance determinants.

Due to sequence quality (lengths of nucleotides with adequate $\mathrm{Tm}$ ) and target homology problems, the target encoding the bifunctional enzyme $\mathrm{AAC}\left(6^{\prime}\right)-\mathrm{Ie}-\mathrm{APH}\left(2^{\prime \prime}\right)-$ Ia conferring HLGR was not represented on the array. The presence of the $\operatorname{aac}\left(6^{\prime}\right)-\operatorname{Ie}-\operatorname{aph}\left(2^{\prime \prime}\right)-I a$ gene was therefore analyzed by PCR and yielded $11 / 20$ and $9 / 20$ of E. faecium and E. faecalis positive for this gene, respectively. Notably, 7/10 E. faecalis ST6 while only two E. faecalis ST40 were positive for aac(6')-Ie-aph(2")-Ia. A strong correlation between the presence of aac (6')-Ieaph(2")-Ia gene and HLGR was observed (data not shown). This observation is consistent with a recent study of European E. faecalis strains that showed a high prevalence of HLGR in CC2 (96\%) in contrast to CC40 (5\%) strains [13].

Determinants of tetracycline resistance were represented by five targets ( 243 for tet $M$; 242 for tetL; 262 for tetK; 385 for tetO; 386 for tetS). Using the CLSI clinical breakpoints, a total of 29/40 strains expressed tetracycline resistance (data not shown). Hybridization analyses revealed that all tetracycline resistant strains harbored one; two or three tet-resistance determinants (see Additional file 3: Figure S2C and Additional file 5: Figure S3C for details). Only $2 / 11$ tetracycline susceptible strains carried tet-resistance determinants (tet $M$ and tet $L$ ), of which both were E. faecium ST78 strains. Interestingly, all E. faecalis strains expressed tetracycline resistance and were shown to carry tet $M(\mathrm{n}=20)$, tetL $(\mathrm{n}=4)$ or tetK $(\mathrm{n}=4)$. Thus, there is a strong correlation between the presence of $\operatorname{Tn} 916 /$ Tn1545 targets and tet $M$ resistance determinants in $E$. faecalis. This observation is in contrast with the findings obtained for E. faecium where eight tetM positive strains were negative for Tn916 xis and int targets. This is even more pronounced than the data from Polish VRE E. faecium isolates where 59 of 72 isolates containing tet $M$ also were positive for the Tn916 integrase gene [42]. These observations could be explained by the location of tet $M$ in other conjugative transposons such as $\operatorname{Tn} 5801$ or Tn6000, frequently found in E. faecium [68]; (Leon-Sampedro, personal communication).

Erythromycin resistance determinants were represented by eight targets ( 260 for ermA; 298 for ermB; 398 for ermF; 399 for ermG; 401 for ermQ; 396 for ermT; 402 for ermTR; 309 for mef). A total of 31/40 and $8 / 40$ strains showed resistance or intermediate susceptibility to erythromycin using the CLSI clinical breakpoints for interpretation (data not shown). Hybridization analyses revealed that all erythromycin resistant strains harbored one of these erythromycin resistance determinants $(\operatorname{erm} B \mathrm{n}=29$; $\operatorname{ermT} \mathrm{n}=1 ;$ mef $\mathrm{n}=1$ ). One susceptible strain was positive for ermB. Determinants ermA, ermF, ermG, and ermQ were not detected. Four out of 8 intermediate strains did not contain any erythromycin resistance determinants, while the other four were positive for $\operatorname{ermB}(\mathrm{n}=1)$, mef $(\mathrm{n}=2)$ or $m e f+\operatorname{ermT}(\mathrm{n}=1)$. One strain was susceptible and also negative for all erythromycin resistance determinants. Acquired resistance towards $M_{L} S_{B}$ in enterococci is most often due to the presence of the ermB gene [121-126]. ermB was identified in $17 / 20 \mathrm{E}$. faecium strains as well as 10/10 and 3/10 E. faecalis ST6 and ST40 strains, respectively (Additional file 5: Figure S3C). Although ermB was originally described as part of Tn917 in E. faecalis [127] we do not find a correlation between presence of ermB and Tn917 targets that were found only in 1 (resolvase target 229) and 2 (transposase target 145) of the 10 ST6 E. faecalis strains. However, ermB presence have been reported on different plasmids [114,128-131] suggesting that ermB is transferred by plasmids among enterococci.

Several chloramphenicol acetyltransferases of different origins were represented on the array (targets 387-393; Additional file 4: Table S2). Target 390 scored positive in $5 / 20$ E. faecalis and 3/20 E. faecium strains, while target 387 scored positive in 9/20 E. faecalis strains (Additional file 5: Figure S3C). Antimicrobial susceptibility testing towards chloramphenicol was not performed. Finally, an oxazolidinone target (target 394) representing a gene encoding rRNA methylase Cfr from a Staphylococcus warneri plasmid was present on the array and one $E$. faecalis strain ST40 scored positive for this target. However, the strain was susceptible to linezolid, also PCR analysis did not detect the $c f r$ gene (data not shown) indicating unspecific hybridization. 
When summarizing the relative presence of the different antimicrobial resistance determinants $(n=67)$ E. faecalis ST40 strains had the highest positive score (average of $10.5 \%$ positive targets per strain) as compared to $E$. faecium ST17, E. faecium ST78 and E. faecalis ST6 with an average of $6.6 \%, 6.9 \%$, and $7.4 \%$, respectively (Additional file 3: Figure $\mathrm{S} 2 \mathrm{C}$ ). The tet $M$, tet $S$ and $m s r C$ genes, responsible for tetracycline and erythromycin resistance, respectively, contributed largely to this difference (Additional file 5: Figure S3C).

\section{High prevalence of genetic determinants for cadmium and QAC resistance in $E$. faecalis ST40}

While the selected targets representing resistance traits towards copper (406 for tcr $B$ ), mercury (407 for merA and 408 for $m e r B$ ), cadmium (409 for $\operatorname{cad} A$ ), and quaternary ammonium compounds (QAC) (410 for qacA, 412 for $s m r / q a c D, 413$ for $q a c G, 414$ for $q a c H$ and 415 for $q a c$ ) seem uncommon in the E. faecium strain collection, a number of $E$. faecalis and in particular ST40 strains hybridized to them (see Additional file 3: Figure S2C and Additional file 5: Figure S3C for details). Hybridization occurred mainly in the strains most recently recovered (2006-2009, Additional file 3: Figure S2C). The cadmium and QAC resistance determinants were found in the same 6/10 E. faecalis ST40 strains as well as in 1/10 ST6 strain but the possible genetic linkage of these resistance determinants will have to be investigated by other methods.

Biocide (e.g., alcohol, aldehyde, biguanides (chlorhexidine), quartenary ammonium compounds, zinc, phenolic compounds) resistance has been rarely detected among enterococcal strains, despite its frequent detection in clinical isolates of other Firmicutes, e.g. the emergence of QAC resistance in Methicillin Resistant Staphylococcus aureus due to acquisition of plasmid-borne qac genes [132,133]. Further, evidence suggests that biocide and antibiotic resistance determinants are linked within the same genetic unit raising the question if the use of biocides can contribute to the selection of antimicrobial resistance [134-139]. However, the previous observed co-localization of the copper resistance determinant with $\operatorname{ermB}[136,137]$ or $\operatorname{vanA}[136]$ in enterococci from animals was not observed in this strain collection representing four highly prevalent, clinical relevant ST types.

\section{Delimited presence of prophage determinants}

Although transduction of virulence and resistance genes were recently demonstrated in enterococci $[139,140]$, the extent and importance of HGT due to bacteriophages in enterococci is not fully understood. Prophage sequences are commonly found in CC2 strains [19] including the fully sequenced $E$. faecalis V583 which harbors seven prophage-like elements [36,141], six of which are proven biologically active [142]. Based on the current knowledge on these sequences at the time the array was printed, five of them (pp1, pp3, pp4, pp6, and pp7) were represented in our assay, Additional file 3: Figure S2D and Additional file 5: Figure S3D. With the exception of one strain from each species, the E. faecalis V583 prophage sequences were present in all E. faecalis test strains, and in none of the E. faecium strains. Prophage7 (pp7) is able to excise from the chromosome by the aid of $\mathrm{pp} 1$ in a manner that resemble chromosomal islands, and have therefore been renamed E. faecalis chromosomal island of V583 (EfcIV583) [132]. This target was only found in two E. faecalis ST6 strains. Eight different E. faecium prophage sequences (24 target sequences of various origins both non-hospital and clinical strains) were represented on the array [15]. With one exception (prophage sequences from E. faecium E980, targets 315-317), these sequences showed species specificity toward E. faecium. Prophage sequences from the clinical E. faecium ST78 strain U0317 (targets 336-341) dominated the E. faecium strain collection and was especially well distributed in the ST78 strains.

Recent studies have identified additional phage sequences to those included in this array (reviewed in [143]) and one should account a possible different outcome if these were included. However, prophage sequences are commonly found in our collection of E. faecium and E. faecalis strains and are in general species-specific, suggesting HGT events as well as vertical transmission of these prophage sequences.

\section{Presence of CRISPR-Cas system only in E. faecalis ST40 strains}

The Clustered Regularly Interspaced Short Palindromic Repeat (CRISPR)/CRISPR associated (Cas) system provides immunity against bacteriophage infections and mobile genetic elements in prokaryotes by genetic memory and (RNA guided) DNA interference (reviewed in $[144,145]$ ). The CRISPR-Cas system was represented in our array by four $E$. faecalis OG1RF CRISPR associated proteins (encoded by cas- and csn genes; targets 474-477) and two conserved CRISPR repeat sequences; one from E. faecium (target 478) and E. faecalis OG1RF (targets 479). None of the 20 E. faecium strains was positive for any of the CRISPR-cas targets (Additional file 3: Figures S2E and Additional file 5: Figure $\mathrm{S} 3 \mathrm{E})$. This is in agreement with previous reports on hospital adapted $E$. faecium lineages with a broad content of antibiotic resistance traits [146] and prophage sequences [15]. This inverse correlation was also expected for the E. faecalis strains [146,147]; however $90 \%$ of the ST40 strains contained both CRISPR and cas sequences, and thus likely a functional system. All these strains also contained multiple acquired antibiotic resistance genes challenging the hypothesis of Palmer and Gilmore [146] that multidrug resistant enterococci generally lack CRISPR-cas genes. A similar 
observation was found in 15 multidrug resistant whole genome sequenced $E$. faecalis ST40 isolates showing supposedly complete CRISPR-Cas systems [148]. It is, however, difficult to elucidate any possible targets for this CRISPR-Cas system without sequencing the CRISPR spacer region to search for specific target sequences.

\section{Conclusions}

Our microarray-based study revealed an overall high prevalence and a species-specific distribution of mobile genetic elements in the selected clinically relevant $E$. faecium and E. faecalis. For the E. faecalis strains, the presence or absence of these targets also separated the two STs. The separation of E. faecalis ST6 and ST40 was especially evident for the biocide genes, E. faecalis prophage sequences and cas genes.

Although the presence of broad host range conjugative Inc18 family replicons substantiates the possibility of inter-species horizontal gene transfer, both species were dominated by narrow host range replicons. The high species-specific prevalence of the RCR family of small cryptic plasmids, the Rep_3 family and the RepA_N family including pLG1 megaplasmids and pheromone responsive plasmids imply an evolutionary significant role in the development of the hospital associated STs hosting them.

An association between the $\omega-\varepsilon-\zeta$ plasmid addiction system and Inc18 plasmids was verified for E. faecium, but the TA locus was also detected in the absence of this plasmid family in both species. The contribution of TA-systems for stable maintenance of important virulence and resistance carrying plasmids in Enterococcus is also underlined by the high prevalence of the axe-txe locus and its association to the RepA_N plasmids in the E. faecium strains.

Specific IS elements are distributed in only one of the species implying that these have evolved vertically. However, the presence of most IS families in both specific strains E. faecalis and E. faecium suggest that these elements are also spread by HGT. Within E. faecium, Tn916/Tn1545 and IS1380 were specific for ST17 and ST78, respectively. All 20 E. faecalis strains contained sequences of $\operatorname{Tn} 916 / \operatorname{Tn} 1545$, while IS families of IS110, IS982 and IS1182 showed a preference towards ST6. The relative lack of $\mathrm{Tn} 916$ family conjugative transposons in E. faecium compared to E. faecalis in our strains fits with most reports of this transposon family in $E$. faecalis. There was also a strong correlation between the presence of Tn916 targets and tetM in E. faecalis that was not found in the E. faecium strains. The presence of Tn917 resolvase and transposase in only one and two of the 30 strains that harbored $\operatorname{ermB}$ correlates well with few descriptions of $\mathrm{Tn} 917$ containing enterococci in the literature although ermB was originally described as part of this transposon in E. faecalis.
The overall findings confirm and unfold our previous knowledge on the extensive reservoir of mobile genetic elements and underline their importance in evolutionary development of clinical relevant lineages of enterococci. Moreover, the detected MGE pattern support a significant species- and for E. faecalis, even a ST-based evolution rather than a high degree of HGT between these important hospital-associated clades.

\section{Availability of supporting data}

The microarray data were deposited in the NCBI Gene Expression Omnibus (GEO) database (http://www.ncbi.nlm. nih.gov/geo/) under accession number GSE59190.

\section{Consent}

The strains are not original samples, but pre-selected, diagnosed and isolated bacterial cultures and as such informed consent from patients is not required. Further, the strains were either given to the co-authors without Material Transfer Agreement (MTA) from originating sources, or given to the co-authours under MTA with the permission to be used in characterization of MGEs. Thus further consent is not necessary.

\section{Additional files}

Additional file 1: Table S1. Correlation matrix for quantile normalized technical replicate (E. faecalis V583). Each fourplex microarray slide included the control strain, E. faecalis V583 to monitor the correlation between the hybridizations and to produce an appropriate cut off value. The overall correlation between the hybridization was 0.95 , producing less than 1/20 false positive hybridizations. The grey rows and columns indicate (number of slide)_(sector on slide)_(number of hybridizations of slide) representing each sector hybridized with genomic DNA of $E$. faecalis $\mathrm{V} 583$.

Additional file 2: Figure S1. A: PCA of the mobilome in 41 enterococcal strains. Each dot represents one of the 41 strains projected by their overall hybridization profile. E. faecium ST17 and ST78 strains are represented by blue and orange, respectively, E. faecalis ST6 and ST40 strains by red and green, respectively, while E. faecium ST92 is marked in purple. Strains are numbered as in Table 1. Each repeated hybridization of control strain E. faecalis V583 is represented by a black spot, B: Cluster dendrogram of the mobilome in 41 enterococcal strains. The dendrogram is visualizing the result of a hierarchical clustering calculation of the mobilome. Strains are numbered as in Table 1, and every application of the control strains $E$. faecalis V583 is indicated by a ' $C$ '.

Additional file 3: Figure S2. Gene profile of the 40 clinical enterococcal strains. Hybridization results of 278 targets grouped into A: plasmid backbone determinants; B: transposable elements; C: resistance determinants; D: phage sequences; and E: CRISPR-Cas sequences in E. faecium ST $17(n=10)$, ST78 $(n=10)$ and ST92 $(n=1)$ and E. faecalis ST6 $(n=10)$ and ST40 $(n=10)$. Positive hybridizations are indicated by black boxes, no hybridization by white boxes.

Additional file 4: Table S2. List of microarray targets and their corresponding probe sequence(s). The acc.no refers to the National Center for Biotechnology Information (NCBI) database (http:// www.ncbi.nlm.nih.gov/), and the target names correspond to the locus names annotated in GenBank. Each target is represented by 1-5 probes in the DNA array as indicated. 
Additional file 5: Figure S3. Summarized gene profile of four enterococcal STs. Number of positive E. faecium ST17 $(n=10)$ and ST78 $(n=10)$ and E. faecalis ST6 $(n=10)$ and ST40 $(n=10)$ for selected targets within five groups; A: plasmid replicons and plasmid addiction systems; B: transposable elements; C: resistance determinants; D: phage; and E: CRISPR-Cas, determined by DNA microarray. Detailed information of each microarray target is found in Additional file 4: Table S2. Note that the presence of pIP501 repR, pCF10 prgW, pLG1 rep, and aac(6)-aph(2")-la are determined by $P C R$. The presence of $\omega-\varepsilon-\zeta$, vanA, vanB, and van $G$ genes are also verified by PCR. Chi square $\left(X^{2}\right)$ calculations are done without corrections, and with 1 degree of freedom using the following online calculator: http://www.graphpad.com/quickcalcs/contingency1.cfm (last accessed December 10th, 2014). Where statistically significant differences $(p<$ 0.05 ) between number of positive strains in each species (E. faecium/E. faecalis) are indicated by light grey (of the species with most positive strains) and between STs (ST17/78 or ST6/40) by dark grey (of the ST with most positive strains). White boxes indicate no significant differences. Verifying PCRs are not included in the calculations.

\section{Competing interests}

The authors declare that they have no competing interests.

\section{Authors' contributions}

TM selected and prepared the targets sequences, collected the strains from the coauthors and hosting laboratory, performed the microarray and PCRs, contributed in analyzing and interpretation of the data and writing the manuscript. TP contributed in the design of the experiment, interpretation of data and substantially contributed to writing the manuscript. RW, TC and GW contributed in the design of the assay, provided target sequences, strains and contributed in writing the manuscript. ES contributed in the design, with strains and in the writing of the manuscript. WVS and LBJ provided targets sequences and contributed in writing the manuscript. AS and $\mathrm{KH}$ contributed in the design of the assay, interpretation and analyzing of the data and writing the manuscript. All authors read and approved the final manuscript.

\section{Authors' information}

Arnfinn Sundsfjord and Kristin Hegstad are joined senior authors.

\section{Acknowledgements}

We thank C. G. Fenton for excellent bioinformatics support and helpful reading of the manuscript, T. M. Lunde for technical assistance, M. V. Francia and P. S. Borra for participation in target selection and preparation, A. M. K. Hammerum and P. Ruiz-Garbajosa for sharing strains.

\begin{abstract}
Author details
'Research group for Host-microbe Interactions, Department of Medical Biology, Faculty of Health Science, UiT - The Arctic University of Norway, Troms $\varnothing$, Norway. ${ }^{2}$ Norwegian National Advisory Unit on Detection of Antimicrobial Resistance, Department of Microbiology and Infection Control, University Hospital of North Norway, Tromsø, Norway. ${ }^{3}$ Department of Medical Microbiology, University Medical Center Utrecht, Utrecht, The Netherlands. ${ }^{4}$ Servicio de Microbiologia, Hospital Ramón y Cajal, Instituto Ramón y Cajal de Investigación Sanitaria (IRYCIS), Madrid, Spain. ${ }^{5}$ Centro de Investigación Biomédica en Red de Epidemiología y Salud Pública (CIBER-ESP), Madrid, Spain. ${ }^{6}$ Division of Nosocomial Pathogens and Antibiotic Resistance, Robert Koch Institute, Wernigerode Branch, Wernigerode, Germany. ${ }^{7}$ Department of Molecular Microbiology, National Medicines Institute, ul, Chełmska 30/34, 00-725 Warsaw, Poland. ${ }^{8}$ Division of Food Microbiologyt, National Food Institute, Danish Technical University, Copenhagen V, Denmark.
\end{abstract}

Received: 11 December 2014 Accepted: 27 February 2015 Published online: 10 April 2015

\section{References}

1. Palmer KL, Kos VN, Gilmore MS. Horizontal gene transfer and the genomics of enterococcal antibiotic resistance. Curr Opin Microbiol. 2010;13(5):632-9.

2. Hegstad K, Mikalsen T, Coque TM, Werner G, Sundsfjord A. Mobile genetic elements and their contribution to the emergence of antimicrobial resistant Enterococcus faecalis and Enterococus faecium. Clin Microbiol Infect. 2010;16(6):451-554.
3. Manson JM, Hancock LE, Gilmore MS. Mechanism of chromosomal transfer of Enterococcus faecalis pathogenicity island, capsule, antimicrobial resistance, and other traits. Proc Natl Acad Sci U S A. 2010;107(27):12269-74.

4. Zhang X, Top J, de Been M, Bierschenk D, Rogers M, Leendertse M, et al. Identification of a genetic determinant in clinical Enterococcus faecium strains that contributes to intestinal colonization during antibiotic treatment. J Infect Dis. 2013;207(11):1780-6.

5. Hendrickx AP, van Schaik W, Willems RJ. The cell wall architecture of Enterococcus faecium: from resistance to pathogenesis. Future Microbiol. 2013;8(8):993-1010.

6. Lebreton F, van Schaik W, McGuire AM, Godfrey P, Griggs A, Mazumdar V, et al. Emergence of epidemic multidrug-resistant Enterococcus faecium from animal and commensal strains. MBio. 2013;20(4(4)):e00534-13.

7. Inoue T, Tomita H, Ike Y. Bac 32, a novel bacteriocin widely disseminated among clinical isolates of Enterococcus faecium. Antimicrob Agents Chemother. 2006;50(4):1202-12.

8. Todokoro D, Tomita H, Inoue T, Ike Y. Genetic analysis of bacteriocin 43 of vancomycin-resistant Enterococcus faecium. Appl Environ Microbiol. 2006;72(11):6955-64.

9. Willems RJ, Hanage WP, Bessen DE, Feil EJ. Population biology of Gram-positive pathogens: high-risk clones for dissemination of antibiotic resistance. FEMS Microbiol Rev. 2011;35(5):872-900.

10. Willems RJ, Top J, van Santen M, Robinson DA, Coque TM, Baquero F, et al. Global spread of vancomycin-resistant Enterococcus faecium from distinct nosocomial genetic complex. Emerg Infect Dis. 2005;11:821-8.

11. Galloway-Pena J, Roh JH, Latorre M, Qin X, Murray BE. Genomic and SNP analyses demonstrate a distant separation of the hospital and communityassociated clades of Enterococcus faecium. PLoS One. 2012;7(1):e30187.

12. Palmer KL, Godfrey P, Griggs A, Kos VN, Zucker J, Desjardins C, et al. Comparative genomics of enterococci: variation in Enterococcus faecalis, clade structure in E. faecium, and defining characteristics of E. gallinarum and E. casseliflavus. MBio. 2012;3(1):e00318-11.

13. Kuch A, Willems RJ, Werner G, Coque TM, Hammerum AM, Sundsfjord A, et al. Insight into antimicrobial susceptibility and population structure of contemporary human Enterococcus faecalis isolates from Europe. J Antimicrob Chemother. 2012;67(3):551-8.

14. Leavis HL, Willems RJ, van Wamel WJ, Schuren FH, Caspers MP, Bonten MJ. Insertion sequence-driven diversification creates a globally dispersed emerging multiresistant subspecies of E. faecium. PLoS Pathog. 2007;3:e7.

15. van Schaik W, Top J, Riley DR, Boekhorst J, Vrijenhoek JE, Schapendonk CM, et al. Pyrosequencing-based comparative genome analysis of the nosocomial pathogen Enterococcus faecium and identification of a large transferable pathogenicity island. BMC Genomics. 2010;11:239:239.

16. Kim EB, Marco ML. Non-clinical and clinical Enterococcus faecium but not Enterococcus faecalis have distinct structural and functional genomic features. Appl Environ Microbiol. 2013;80(1):154-65.

17. Rosvoll TC, Pedersen T, Sletvold H, Johnsen PJ, Sollid JE, Simonsen GS, et al. PCR-based plasmid typing in Enterococcus faecium strains reveals widely distributed pRE25-, pRUM-, pIP501- and pHTbeta-related replicons associated with glycopeptide resistance and stabilizing toxin-antitoxin systems. FEMS Immunol Med Microbiol. 2010;58(2):254-68.

18. van Schaik W, Willems RJ. Genome-based insights into the evolution of enterococci. Clin Microbiol Infect. 2010;16:527-32.

19. Solheim M, Brekke MC, Snipen LG, Willems RJ, Nes IF, Brede DA. Comparative genomic analysis reveals significant enrichment of mobile genetic elements and genes encoding surface structure-proteins in hospital-associated clonal complex 2 Enterococcus faecalis. BMC Microbiol. 2011;11:3.

20. Lam MM, Seemann T, Bulach DM, Gladman SL, Chen H, Haring V, et al. Comparative analysis of the first complete Enterococcus faecium genome. J Bacteriol. 2012;194(9):2334-41.

21. Freitas AR, Novais C, Tedim AP, Francia MV, Baquero F, Peixe L, et al. Microevolutionary events involving narrow host plasmids influences local fixation of vancomycin-resistance in enterococcus populations. PLoS One. 2013;8(3):e60589.

22. Werner G, Coque TM, Franz CM, Grohmann E, Hegstad K, Jensen L, et al. Antibiotic resistant enterococci-Tales of a drug resistance gene trafficker. Int J Med Microbiol. 2013;303(6-7):360-79.

23. Coque TM. Evolutionary Biology of Pathogenic Enterococci. In: Baquero F, Nombela C, Cassell GH, et al., editors. Evolutionary Biology of Bacterial and Fungal Pathogens. 2008. 
24. Jensen LB, Garcia-Migura L, Valenzuela AJ, Lohr M, Hasman H, Aarestrup FM. A classification system for plasmids from enterococci and other Grampositive bacteria. J Microbiol Methods. 2010;80(1):25-43.

25. Freitas AR, Tedim AP, Novais C, Ruiz-Garbajosa P, Werner G, Laverde-Gomez $J A$, et al. Global spread of the hyl(Efm) colonization-virulence gene in megaplasmids of the Enterococcus faecium CC17 polyclonal subcluster. Antimicrob Agents Chemother. 2010;54:2660-5.

26. Wardal E, Gawryszewska I, Hryniewicz W, Sadowy E. Abundance and diversity of plasmid-associated genes among clinical isolates of Enterococcus faecalis. Plasmid. 2013;70(3):329-42.

27. Quintiliani Jr R, Courvalin P. Conjugal transfer of the vancomycin resistance determinant vanB between enterococci involves the movement of large genetic elements from chromosome to chromosome. FEMS Microbiol Lett. 1994;119(3):359-63.

28. Moritz EM, Hergenrother PJ. Toxin-antitoxin systems are ubiquitous and plasmid-encoded in vancomycin-resistant enterococci. Proc Natl Acad Sci U S A. 2007;104:311-6.

29. Rosvoll TC, Lindstad BL, Lunde TM, Hegstad K, Aasnaes B, Hammerum AM, et al. Increased high-level gentamicin resistance in invasive Enterococcus faecium is associated with aac $\left(6^{\prime}\right)$ le-aph $\left(2^{\prime \prime}\right)$ la-encoding transferable megaplasmids hosted by major hospital-adapted lineages. FEMS Immunol Med Microbiol. 2012;66(2):166-76.

30. Hayes F, van Melderen L. Toxins-antitoxins: diversity, evolution and function. Crit Rev Biochem Mol Biol. 2011;46(5):386-408.

31. Mutschler $\mathrm{H}$, Meinhart A. epsilon/zeta systems: their role in resistance, virulence, and their potential for antibiotic development. J Mol Med (Berl). 2011;89(12):1183-94.

32. Unterholzner SJ, Poppenberger B, Rozhon W. Toxin-antitoxin systems: Biology, identification, and application. Mob Genet Elements. 2013;3(5):e26219.

33. Schuster CF, Bertram R. Toxin-antitoxin systems are ubiquitous and versatile modulators of prokaryotic cell fate. FEMS Microbiol Lett. 2013;340(2):73-85.

34. Hayes F, Kedzierska B. Regulating toxin-antitoxin expression: controlled detonation of intracellular molecular timebombs. Toxins (Basel). 2014;6(1):337-58.

35. Siefert JL. Defining the mobilome. Methods Mol Biol. 2009;532:13-27.

36. Paulsen IT, Banerjei L, Myers GS, Nelson KE, Seshadri R, Read TD, et al. Role of mobile DNA in the evolution of vancomycin-resistant Enterococcus faecalis. Science. 2003;299:2071-4.

37. Top J, Willems R, Bonten M. Emergence of CC17 Enterococcus faecium: from commensal to hospital-adapted pathogen. FEMS Immunol Med Microbiol. 2008;52:297-308.

38. Dahl KH, Simonsen GS, Olsvik O, Sundsfjord A. Heterogeneity in the vanB gene cluster of genomically diverse clinical strains of vancomycin-resistant enterococci. Antimicrob Agents Chemother. 1999;43:1105-10.

39. Dahl KH, Rokenes TP, Lundblad EW, Sundsfjord A. Nonconjugative transposition of the vanB-containing Tn5382-like element in Enterococcus faecium. Antimicrob Agents Chemother. 2003:47:786-9.

40. Coque TM, Ruíz-Garbajosa P, Tedim AP, Freitas AR, Baquero F, Bou G, et al Estructura poblacional de Enterococcus faecium resistente a vancomicina (EfmRV) aislados en hospitales españoles (1996-2010). Barcelona, Spain: XIV Congreso SEIMC; 2010. Ref Type: Abstract.

41. Bjorkeng E, Rasmussen G, Sundsfjord A, Sjoberg L, Hegstad K, Soderquist B. Clustering of polyclonal VanB-type vancomycin-resistant Enterococcus faecium in a low-endemic area was associated with CC17-genogroup strains harbouring transferable vanB2-Tn5382 and pRUM-like repA containing plasmids with axe-txe plasmid addiction systems. APMIS. 2011;119(4-5):247-58.

42. Sadowy E, Sienko A, Gawryszewska I, Bojarska A, Malinowska K, Hryniewicz W. High abundance and diversity of antimicrobial resistance determinants among early vancomycin-resistant Enterococcus faecium in Poland. Eur J Clin Microbiol Infect Dis. 2013;32(9):1193-203.

43. Lester $\mathrm{CH}$, Olsen SS, Schonheyder HC, Hansen DS, Tvede M, Holm A, et al. Typing of vancomycin-resistant enterococci obtained from patients at Danish hospitals and detection of a genomic island specific to CC17 Enterococcus faecium. Int J Antimicrob Agents. 2010;35:312-4.

44. Werner G, Fleige C, Ewert B, Laverde-Gomez JA, Klare I, Witte W. High-level ciprofloxacin resistance among hospital-adapted Enterococcus faecium (CC17). Int J Antimicrob Agents. 2010;35(2):119-25.

45. Werner G, Fleige C, Geringer U, Van SW, Klare I, Witte W. IS element IS16 as a molecular screening tool to identify hospital-associated strains of Enterococcus faecium. BMC Infect Dis. 2011;11:80.

46. Bjorkeng EK, Tessema GT, Lundblad EW, Butaye P, Willems R, Sollid JE, et al. ccrABEnt serine recombinase genes are widely distributed in the Enterococcus faecium and Enterococcus casseliflavus species groups and are expressed in E. faecium. Microbiology. 2010;156(Pt 12):3624-34.

47. Freitas AR, Novais C, Ruiz-Garbajosa P, Coque TM, Peixe L. Dispersion of multidrug-resistant Enterococcus faecium isolates belonging to major clonal complexes in different Portuguese settings. Appl Environ Microbiol. 2009;75:4904-8.

48. Sivertsen A, Billstrom H, Melefors O, Liljequist BO, Wisell KT, Ullberg M, et al. A multicentre hospital outbreak in Sweden caused by introduction of a vanB2 transposon into a stably maintained pRUM-plasmid in an Enterococcus faecium ST192 clone. PLoS One. 2014;9(8):e103274.

49. Novais C, Coque TM, Sousa JC, Baquero F, Peixe L. Local genetic patterns within a vancomycin-resistant Enterococcus faecalis clone isolated in three hospitals in Portugal. Antimicrob Agents Chemother. 2004;48:3613-7.

50. Freitas AR, Coque TM, Novais C, Hammerum AM, Lester CH, Zervos MJ, et al. Human and swine hosts share vancomycin-resistant Enterococcus faecium CC17 and CC5 and Enterococcus faecalis CC2 clonal clusters harboring Tn1546 on indistinguishable plasmids. J Clin Microbiol. 2011;49(3):925-31.

51. Freitas AR, Novais C, Ruiz-Garbajosa P, Coque TM, Peixe L. Clonal expansion within clonal complex 2 and spread of vancomycin-resistant plasmids among different genetic lineages of Enterococcus faecalis from Portugal. J Antimicrob Chemother. 2009;63:1104-11.

52. Ruiz-Garbajosa P, Bonten MJ, Robinson DA, Top J, Nallapareddy SR, Torres C, et al. Multilocus sequence typing scheme for Enterococcus faecalis reveals hospital-adapted genetic complexes in a background of high rates of recombination. J Clin Microbiol. 2006;44:2220-8.

53. Zischka M, Fleige C, Günther C, Klare I, Witte W, Werner G. Comparative genomics of Enterococcus faecalis ST40 - Differences between commensal and clinical isolates. 9th IMMEM, Wernigerode, Germany, Abstract V.10. 1-4-2010. Ref Type: Abstract.

54. Zischka M, Dabrowski PW, Fleige C, Schreiber K, Witte W, Werner G. Comparative Genomics of Enterococcus faecalis ST40 - Differences Between Commensal and Clinical Isolates. Göttingen, Germany: 5th European Conference on Prokaryotic and fungal genomics. 2011. Abstract P82. 18-9-2011. Ref Type: Abstract.

55. Kawalec M, Pietras Z, Danilowicz E, Jakubczak A, Gniadkowski M, Hryniewicz W, et al. Clonal structure of Enterococcus faecalis isolated from Polish hospitals: characterization of epidemic clones. J Clin Microbiol. 2007:45:147-53.

56. Sahm DF, Kissinger J, Gilmore MS, Murray PR, Mulder R, Solliday J, et al. In vitro susceptibility studies of vancomycin-resistant Enterococcus faecalis. Antimicrob Agents Chemother. 1989;33:1588-91.

57. Vakulenko SB, Donabedian SM, Voskresenskiy AM, Zervos MJ, Lerner SA, Chow JW. Multiplex PCR for detection of aminoglycoside resistance genes in enterococci. Antimicrob Agents Chemother. 2003;47(4):1423-6.

58. Patel R, Uhl JR, Kohner P, Hopkins MK, Steckelberg JM, Kline B, et al. DNA sequence variation within $\operatorname{van} A$, vanB, vanC -1 , and vanC $-2 / 3$ genes of clinical Enterococcus isolates. Antimicrob Agents Chemother. 1998;42(1):202-5.

59. Willems RJ, Top J, van den Braak N, van Belkum A, Mevius DJ, Hendriks G, et al. Molecular diversity and evolutionary relationships of Tn1546-like elements in enterococci from humans and animals. Antimicrob Agents Chemother. 1999:43:483-91.

60. Laverde Gomez JA, Van SW, Freitas AR, Coque TM, Weaver KE, Francia MV, et al. A multiresistance megaplasmid pLG1 bearing a hylEfm genomic island in hospital Enterococcus faecium isolates. Int J Med Microbiol. 2011;301(2):165-75.

61. The European Committee on Antimicrobial Susceptibility Testing. EUCAST Disk Diffusion Test Manual.v.3.0,2013. Available at http://www.eucast.org (last accessed December 10th,2014). EUCAST 2013.

62. Swenson JM, Clark NC, Ferraro MJ, Sahm DF, Doern G, Pfaller MA, et al. Development of a standardized screening method for detection of vancomycin-resistant enterococci. J Clin Microbiol. 1994;32(7):1700-4.

63. The European Committee on Antimicrobial Susceptibility Testing: Breakpoints tables for interpretation of MICs and zone diameters.v.3.0,2013 Available at http://www.eucast.org (last accessed December 10th,2014). v 3.0, 2013. EUCAST 2013.

64. Willems RJ, Top J, van Schaik W, Leavis H, Bonten M, Siren J, et al. Restricted gene flow among hospital subpopulations of Enterococcus faecium. MBio. 2012;3(4):e00151-12.

65. Salzberg SL, Phillippy AM, Zimin A, Puiu D, Magoc T, Koren S, et al. GAGE: A critical evaluation of genome assemblies and assembly algorithms. Genome Res. 2012;22(3):557-67.

66. Junemann S, Sedlazeck FJ, Prior K, Albersmeier A, John U, Kalinowski J, et al. Updating benchtop sequencing performance comparison. Nat Biotechnol. 2013;31(4):294-6. 
67. Junemann S, Prior K, Albersmeier A, Albaum S, Kalinowski J, Goesmann A, et al. GABenchToB: a genome assembly benchmark tuned on bacteria and benchtop sequencers. PLoS One. 2014;9(9):e107014.

68. Clewell DB, Weaver KE, Dunny GM, Coque TM, Francia MV, Hayes F. Extrachromosomal and Mobile Elements in Enterococci: Transmission, Maintenance, and Epidemiology. In: Gilmore MS, Clewell DB, Ike Y, et al., editors. Enterococci: From Commensals to Leading Causes of Drug Resistant Infection. Boston: Massuchusetts Eye and Ear Infirmary; 2014.

69. Garcia-Migura L, Hasman H, Jensen LB. Presence of pRI1: a small cryptic mobilizable plasmid isolated from Enterococcus faecium of human and animal origin. Curr Microbiol. 2009;58:95-100.

70. Ridenhour MB, Fletcher HM, Mortensen JE, Daneo-Moore L. A novel tetracycline-resistant determinant, tet(U), is encoded on the plasmid pKq10 in Enterococcus faecium. Plasmid. 1996:35(2):71-80.

71. Chen YG, Qu TT, Yu YS, Zhou JY, Li LJ. Insertion sequence ISEcp 1-like element connected with a novel aph( $\left.2^{\prime \prime}\right)$ allele [aph( $\left.2^{\prime \prime}\right)$-le] conferring high-level gentamicin resistance and a novel streptomycin adenylyltransferase gene in Enterococcus. J Med Microbiol. 2006;55:1521-5.

72. Wyckoff HA, Barnes M, Gillies KO, Sandine WE. Characterization and sequence analysis of a stable cryptic plasmid from Enterococcus faecium 226 and development of a stable cloning vector. Appl Environ Microbiol. 1996;62(4):1481-6.

73. Criado R, Gutierrez J, Budin-Verneuil A, Hernandez PE, Hartke A, Cintas LM, et al. Molecular analysis of the replication region of the $\mathrm{pCI} 22$ plasmid from the multiple bacteriocin producer strain Enterococcus faecium L50. Plasmid. 2008;60(3):181-9.

74. Martinez-Bueno M, Valdivia E, Galvez A, Maqueda M. pS86, a new theta-replicating plasmid from Enterococcus faecalis. Curr Microbiol. 2000;41(4):257-61.

75. Balla E, Dicks LM. Molecular analysis of the gene cluster involved in the production and secretion of enterocins 1071A and 1071B and of the genes responsible for the replication and transfer of plasmid pEF1071. Int J Food Microbiol. 2005;99(1):33-45.

76. Weaver KE, Kwong SM, Firth N, Francia MV. The RepA_N replicons of Gram-positive bacteria: a family of broadly distributed but narrow host range plasmids. Plasmid. 2009;61:94-109.

77. Hirt H, Manias DA, Bryan EM, Klein JR, Marklund JK, Staddon JH, et al Characterization of the pheromone response of the Enterococcus faecalis conjugative plasmid pCF10: complete sequence and comparative analysis of the transcriptional and phenotypic responses of pCF10-containing cells to pheromone induction. J Bacteriol. 2005;187(3):1044-54.

78. Weaver KE, Clewell DB, An F. Identification, characterization, and nucleotide sequence of a region of Enterococcus faecalis pheromoneresponsive plasmid pAD1 capable of autonomous replication. J Bacteriol. 1993;175:1900-9.

79. Fujimoto S, Tomita H, Wakamatsu E, Tanimoto K, Ike Y. Physical mapping of the conjugative bacteriocin plasmid PPD1 of Enterococcus faecalis and identification of the determinant related to the pheromone response. J Bacteriol. 1995;177(19):5574-81.

80. Weaver KE, Reddy SG, Brinkman CL, Patel S, Bayles KW, Endres JL. Identification and characterization of a family of toxin-antitoxin systems related to the Enterococcus faecalis plasmid pAD1 par addiction module. Microbiology. 2009;155(Pt 9):2930-40.

81. Grady R, Hayes F. Axe-Txe, a broad-spectrum proteic toxin-antitoxin system specified by a multidrug-resistant, clinical isolate of Enterococcus faecium. Mol Microbiol. 2003:47:1419-32.

82. Zhu W, Murray PR, Huskins WC, Jernigan JA, McDonald LC, Clark NC, et al. Dissemination of an Enterococcus Inc18-Like vanA plasmid associated with vancomycin-resistant Staphylococcus aureus. Antimicrob Agents Chemother. 2010;54(10):4314-20.

83. Sletvold H, Johnsen PJ, Simonsen GS, Aasnaes B, Sundsfjord A, Nielsen KM. Comparative DNA analysis of two vanA plasmids from Enterococcus faecium strains isolated from poultry and a poultry farmer in Norway. Antimicrob Agents Chemother. 2007;51:736-9.

84. Sletvold H, Johnsen PJ, Hamre I, Simonsen GS, Sundsfjord A, Nielsen KM. Complete sequence of Enterococcus faecium pVEF3 and the detection of an omega-epsilon-zeta toxin-antitoxin module and an $A B C$ transporter. Plasmid. 2008;60(1):75-85.

85. Zielenkiewicz U, Ceglowski P. The toxin-antitoxin system of the streptococcal plasmid pSM19035. J Bacteriol. 2005;187:6094-105.

86. Mittenhuber G. Occurrence of mazEF-like antitoxin/toxin systems in bacteria. J Mol Microbiol Biotechnol. 1999;1(2):295-302.
87. Bertram R, Schuster CF. Post-transcriptional regulation of gene expression in bacterial pathogens by toxin-antitoxin systems. Front Cell Infect Microbiol. 2014:4:6.

88. Christie PJ, Dunny GM. Identification of regions of the Streptococcus faecalis plasmid pCF-10 that encode antibiotic resistance and pheromone response functions. Plasmid. 1986;15(3):230-41.

89. Clewell DB, An FY, White BA, Gawron-Burke C. Streptococcus faecalis sex pheromone (CAM373) also produced by Staphylococcus aureus and identification of a conjugative transposon (Tn918). J Bacteriol. 1985;162(3):1212-20.

90. Hodel-Christian SL, Murray BE. Characterization of the gentamicin resistance transposon Tn5281 from Enterococcus faecalis and comparison to staphylococcal transposons Tn4001 and Tn4031. Antimicrob Agents Chemother. 1991;35(6):1147-52.

91. Quintiliani Jr R, Courvalin P. Characterization of Tn1547, a composite transposon flanked by the IS16 and IS256-like elements, that confers vancomycin resistance in Enterococcus faecalis BM4281. Gene. 1996;172:1-8.

92. Dahl KH, Lundblad EW, Rokenes TP, Olsvik O, Sundsfjord A. Genetic linkage of the vanB2 gene cluster to Tn5382 in vancomycin-resistant enterococci and characterization of two novel insertion sequences. Microbiology. 2000;146:1469-79.

93. Rice LB, Carias LL, Marshall SH. Tn5384, a composite enterococcal mobile element conferring resistance to erythromycin and gentamicin whose ends are directly repeated copies of IS256. Antimicrob Agents Chemother. 1995;39(5):1147-53.

94. Darini AL, Palepou MF, Woodford N. Effects of the movement of insertion sequences on the structure of VanA glycopeptide resistance elements in Enterococcus faecium. Antimicrob Agents Chemother. 2000;44(5):1362-4

95. Cheng SQ, Musso RE, Liu R, Niemeyer DM, Shaw JJ, McCleskey FK, et al. Cloning of insertion sequence IS1485 from Enterococcus species. Plasmid. 1999;42:42-4.

96. Lee WG, Kim W. Identification of a novel insertion sequence in vanB2containing Enterococcus faecium. Lett Appl Microbiol. 2003;36:186-90.

97. Handwerger S, Skoble J, Discotto LF, Pucci MJ. Heterogeneity of the vanA gene cluster in clinical isolates of enterococci from the northeastern United States. Antimicrob Agents Chemother. 1995;39:362-8.

98. Simonsen GS, Myhre MR, Dahl KH, Olsvik O, Sundsfjord A. Typeability of Tn1546-like elements in vancomycin-resistant enterococci using long-range PCRs and specific analysis of polymorphic regions. Microb Drug Resist. 2000;6:49-57.

99. Camargo IL, Del Peloso PF, Da Costa Leite CF, Goldman GH, Darini AL. Identification of an unusual VanA element in glycopeptide-resistant Enterococcus faecium in Brazil following international transfer of a bone marrow transplant patient. Can J Microbiol. 2004;50:767-70.

100. Lopez M, Saenz Y, Alvarez-Martinez MJ, Marco F, Robredo B, Rojo-Bezares B, et al. Tn1546 structures and multilocus sequence typing of vanA-containing enterococci of animal, human and food origin. J Antimicrob Chemother. 2010;65(8):1570-5.

101. MacKinnon MG, Drebot MA, Tyrrell GJ. Identification and characterization of IS1476, an insertion sequence-like element that disrupts VanY function in a vancomycin-resistant Enterococcus faecium strain. Antimicrob Agents Chemother. 1997:41:1805-7.

102. Depardieu F, Reynolds PE, Courvalin P. VanD-type vancomycin-resistant Enterococcus faecium 10/96A. Antimicrob Agents Chemother. 2003;47:7-18.

103. Gu L, Cao B, Liu Y, Guo P, Song S, Li R, et al. A new Tn1546 type of VanB phenotype-vanA genotype vancomycin-resistant Enterococcus faecium isolates in mainland China. Diagn Microbiol Infect Dis. 2009;63(1):70-5.

104. Camargo IL, Zanella RC, Brandileone MC, Pignatari AC, Goldman GH, Woodford N, et al. Occurrence of insertion sequences within the genomes and Tn1546-like elements of glycopeptide-resistant enterococci isolated in Brazil, and identification of a novel element, ISEfa5. Int J Med Microbiol. 2005;294:513-9.

105. Khan MA, Northwood JB, Loor RG, Tholen AT, Riera E, Falcon M, et al. High prevalence of ST-78 infection-associated vancomycin-resistant Enterococcus faecium from hospitals in Asuncion, Paraguay. Clin Microbiol Infect. 2010;16(6):624-7.

106. Boyd DA, Conly J, Dedier H, Peters G, Robertson L, Slater E, et al. Molecular characterization of the vanD gene cluster and a novel insertion element in a vancomycin-resistant enterococcus isolated in Canada. J Clin Microbiol. 2000:38:2392-4. 
107. Derbise A, De CG, El SN. Nucleotide sequence of the Staphylococcus aureus transposon, Tn5405, carrying aminoglycosides resistance genes. J Basic Microbiol. 1997;37(5):379-84.

108. Werner G, Hildebrandt B, Witte W. Linkage of erm(B) and aadE-sat4-aphA-3 in multiple-resistant Enterococcus faecium isolates of different ecological origins. Microb Drug Resist. 2003;9 Suppl 1:S9-16.

109. Jung WK, Hong SK, Koo HC, Kwon NH, Park YH. Nucleotide sequence of IS1678, an insertion sequence in the vanA cluster of enterococci. Antimicrob Agents Chemother. 2005;49:1666-7.

110. Halvorsen EM, Williams JJ, Bhimani AJ, Billings EA, Hergenrother PJ. Txe, an endoribonuclease of the enterococcal Axe-Txe toxin-antitoxin system, cleaves mRNA and inhibits protein synthesis. Microbiology. 2011;157(Pt 2):387-97.

111. Rice LB, Carias LL, Donskey CL, Rudin SD. Transferable, plasmid-mediated vanB-type glycopeptide resistance in Enterococcus faecium. Antimicrob Agents Chemother. 1998;42(4):963-4.

112. Roberts AP, Mullany P. A modular master on the move: the Tn916 family of mobile genetic elements. Trends Microbiol. 2009;17(6):251-8.

113. Top J, Sinnige JC, Majoor EA, Bonten MJ, Willems RJ, van Schaik W. The recombinase IntA is required for excision of esp-containing ICEEfm1 in Enterococcus faecium. J Bacteriol. 2011;193(4):1003-6.

114. Lim SK, Tanimoto K, Tomita H, Ike Y. Pheromone-responsive conjugative vancomycin resistance plasmids in Enterococcus faecalis isolates from humans and chicken feces. Appl Environ Microbiol. 2006;72:6544-53.

115. Arthur M, Molinas C, Depardieu F, Courvalin P. Characterization of Tn1546, a Tn3-related transposon conferring glycopeptide resistance by synthesis of depsipeptide peptidoglycan precursors in Enterococcus faecium BM4147. J Bacteriol. 1993;175:117-27.

116. Garnier F, Taourit S, Glaser P, Courvalin P, Galimand M. Characterization of transposon Tn1549, conferring VanB-type resistance in Enterococcus spp. Microbiology. 2000;146(Pt 6):1481-9.

117. McGregor KF, Nolan C, Young HK, Palepou MF, Tysall L, Woodford N. Prevalence of the vanB2 gene cluster in vanB glycopeptide-resistant enterococci in the United Kingdom and the Republic of Ireland and its association with a Tn5382-like element. Antimicrob Agents Chemother. 2001;45(1):367-8.

118. Cha JO, Yoo Jl, Kim HK, Kim HS, Yoo JS, Lee YS, et al. Diversity of Tn1546 in vanA-positive Enterococcus faecium clinical isolates with VanA, VanB, and VanD phenotypes and susceptibility to vancomycin. J Appl Microbiol. 2013;115(4):969-76.

119. Coburn B, Low DE, Patel SN, Poutanen SM, Shahinas D, Eshaghi A, et al. Vancomycin variable Enterococcus faecium (WE): in vivo emergence of vancomycin resistance in a vancomycin susceptible isolate. J Clin Microbiol. 2014;52(5):1766-7.

120. Szakacs TA, Kalan L, McConnell MJ, Eshaghi A, Shahinas D, McGeer A, et al. Outbreak of Vancomycin susceptible Enterococcus faecium containing wild-type vanA gene. J Clin Microbiol. 2014;52(5):1682-7.

121. Aarestrup FM, Agerso $Y$, Gerner-Smidt P, Madsen M, Jensen LB. Comparison of antimicrobial resistance phenotypes and resistance genes in Enterococcus faecalis and Enterococcus faecium from humans in the community, broilers, and pigs in Denmark. Diagn Microbiol Infect Dis. 2000;37(2):127-37.

122. De LE, Martel A, De Graef EM, Top J, Butaye P, Haesebrouck F, et al. Molecular analysis of human, porcine, and poultry Enterococcus faecium isolates and their erm(B) genes. Appl Environ Microbiol. 2005;71(5):2766-70.

123. Jensen LB, Frimodt-Moller N, Aarestrup FM. Presence of erm gene classes in gram-positive bacteria of animal and human origin in Denmark. FEMS Microbiol Lett. 1999;170(1):151-8.

124. Portillo A, Ruiz-Larrea F, Zarazaga M, Alonso A, Martinez JL, Torres C. Macrolide resistance genes in Enterococcus spp. Antimicrob Agents Chemother. 2000;44(4):967-71.

125. Roberts MC, Sutcliffe J, Courvalin P, Jensen LB, Rood J, Seppala H. Nomenclature for macrolide and macrolide-lincosamide-streptogramin $\mathrm{B}$ resistance determinants. Antimicrob Agents Chemother. 1999;43(12):2823-30.

126. Weisblum B. Erythromycin resistance by ribosome modification. Antimicrob Agents Chemother. 1995;39(3):577-85.

127. Tomich PK, An FY, Clewell DB. Properties of erythromycin-inducible transposon Tn917 in Streptococcus faecalis. J Bacteriol. 1980;141(3):1366-74.

128. Tremblay $C L$, Letellier $A$, Quessy $S$, Boulianne $M$, Daignault $D$, Archambault M. Multiple-antibiotic resistance of Enterococcus faecalis and Enterococcus faecium from cecal contents in broiler chicken and turkey flocks slaughtered in Canada and plasmid colocalization of tetO and ermB genes. J Food Prot. 2011;74(10):1639-48.
129. Teuber M, Schwarz F, Perreten V. Molecular structure and evolution of the conjugative multiresistance plasmid pRE25 of Enterococcus faecalis isolated from a raw-fermented sausage. Int J Food Microbiol. 2003;88:325-9.

130. Jackson CR, Fedorka-Cray PJ, Barrett JB, Ladely SR. Effects of tylosin use on erythromycin resistance in enterococci isolated from swine. Appl Environ Microbiol. 2004;70(7):4205-10.

131. Werner G, Klare I, Witte W. Molecular analysis of streptogramin resistance in enterococci. Int J Med Microbiol. 2002;292(2):81-94.

132. Mayer S, Boos M, Beyer A, Fluit AC, Schmitz FJ. Distribution of the antiseptic resistance genes qac $A$, qacB and qacC in 497 methicillin-resistant and -susceptible European isolates of Staphylococcus aureus. J Antimicrob Chemother. 2001;47(6):896-7.

133. Noguchi N, Hase M, Kitta M, Sasatsu M, Deguchi K, Kono M. Antiseptic susceptibility and distribution of antiseptic-resistance genes in methicillinresistant Staphylococcus aureus. FEMS Microbiol Lett. 1999;172(2):247-53.

134. Russell AD. Do biocides select for antibiotic resistance? J Pharm Pharmacol. 2000;52(2):227-33.

135. Hegstad K, Langsrud S, Lunestad BT, Scheie AA, Sunde M, Yazdankhah SP. Does the wide use of quaternary ammonium compounds enhance the selection and spread of antimicrobial resistance and thus threaten our health? Microb Drug Resist. 2010;16(2):91-104.

136. Hasman H, Aarestrup FM. tcrB, a gene conferring transferable copper resistance in Enterococcus faecium: occurrence, transferability, and linkage to macrolide and glycopeptide resistance. Antimicrob Agents Chemother. 2002;46:1410-6.

137. Amachawadi RG, Shelton NW, Shi X, Vinasco J, Dritz SS, Tokach MD, et al. Selection of fecal enterococci exhibiting tcrB-mediated copper resistance in pigs fed diets supplemented with copper. Appl Environ Microbiol. 2011;77(16):5597-603.

138. Silveira E, Freitas AR, Antunes P, Barros M, Campos J, Coque TM, et al. Co-transfer of resistance to high concentrations of copper and first-line antibiotics among Enterococcus from different origins (humans, animals, the environment and foods) and clonal lineages. J Antimicrob Chemother. 2014;69(4):899-906.

139. Yasmin A, Kenny JG, Shankar J, Darby AC, Hall N, Edwards C, et al. Comparative genomics and transduction potential of Enterococcus faecalis temperate bacteriophages. J Bacteriol. 2010;192(4):1122-30.

140. Mazaheri Nezhad FR, Barton MD, Heuzenroeder MW. Bacteriophagemediated transduction of antibiotic resistance in enterococci. Lett Appl Microbiol. 2011;52(6):559-64.

141. McBride SM, Fischetti VA, Leblanc DJ, Moellering Jr RC, Gilmore MS. Genetic diversity among Enterococcus faecalis. PLoS One. 2007;2:e582.

142. Matos RC, Lapaque N, Rigottier-Gois L, Debarbieux L, Meylheuc T, GonzalezZorn $B$, et al. Enterococcus faecalis prophage dynamics and contributions to pathogenic traits. PLoS Genet. 2013;9(6):e1003539.

143. Breck A. Duerkop, Palmer KL, Horsburgh MJ. Enterococcal Bacteriophages and Genome Defense. Enterococci: From Commensal to Leading Causes of Drug Resistant Infection. Edited by Gilmore MS, Clewell DB, Ike Y, Shankar N. Boston: Massachusetts Eye and Ear Infirmary; 2014.

144. Bikard D, Marraffini LA. Control of gene expression by CRISPR-Cas systems. F1000Prime Rep. 2013;5:47.

145. Louwen R, Staals RH, Endtz HP, Van BP, van der Oost J. The role of CRISPRCas systems in virulence of pathogenic bacteria. Microbiol Mol Biol Rev. 2014;78(1):74-88.

146. Palmer KL, Gilmore MS. Multidrug-resistant enterococci lack CRISPR-cas. MBio. 2010;1(4):e00227-10.

147. Burley KM, Sedgley CM. CRISPR-Cas, a prokaryotic adaptive immune system, in endodontic, oral, and multidrug-resistant hospital-acquired Enterococcus faecalis. J Endod. 2012;38(11):1511-5.

148. Zischka M, Künne CT, Blom J, Wobser D, Sakınç T, Schmidt-Hohagen K, et al. Comprehensive molecular, genomic and phenotypic analysis of a major clone of Enterococcus faecalis MLST ST40. BMC Genomics 2015;16:175. doi:10.1186/s12864-015-1367-x 